Preprint typeset in JHEP style - HYPER VERSION

\title{
Center vortices and the Gribov horizon
}

\author{
Jeff Greensite \\ The Niels Bohr Institute, Blegdamsvej 17, DK-2100 Copenhagen Ø, Denmark, \\ Physics and Astronomy Dept., San Francisco State University, San Francisco, \\ CA 94117, USA. \\ E-mail: greensit@stars.sfsu.edu

\section{Štefan Olejník} \\ Institute of Physics, Slovak Academy of Sciences, SK-845 11 Bratislava, Slovakia. \\ E-mail: stefan.olejnik@savba.sk

\section{Daniel Zwanziger} \\ Physics Department, New York University, New York, NY 10003, USA. \\ E-mail: daniel.zwanziger@nyu.edu
}

\begin{abstract}
We show how the infinite color-Coulomb energy of color-charged states is related to enhanced density of near-zero modes of the Faddeev-Popov operator, and calculate this density numerically for both pure Yang-Mills and gauge-Higgs systems at zero temperature, and for pure gauge theory in the deconfined phase. We find that the enhancement of the eigenvalue density is tied to the presence of percolating center vortex configurations, and that this property disappears when center vortices are either removed from the lattice configurations, or cease to percolate. We further demonstrate that thin center vortices have a special geometrical status in gauge-field configuration space: Thin vortices are located at conical or wedge singularities on the Gribov horizon. We show that the Gribov region is itself a convex manifold in lattice configuration space. The Coulomb gauge condition also has a special status; it is shown to be an attractive fixed point of a more general gauge condition, interpolating between the Coulomb and Landau gauges.
\end{abstract}

Keywords: Confinement, Lattice Gauge Field Theories, Solitons Monopoles and Instantons. 


\section{Contents}

1. Introduction 2

2. The F-P eigenvalue density 3

2.1 Observables 6

2.2 Center projection and vortex removal 7

2.3 Results 9

3. Gauge-Higgs theory 15

4. Eigenvalue density in the deconfined phase 18

5. Thin vortices and the eigenvalue density 19

6. Convexity of FMR and Gribov regions in SU(2) lattice gauge theory 22

7. Vortices as vertices 24

7.1 Previous results 24

7.2 Tangent plane to Gribov horizon at a regular point 24

7.3 Center vortices as singularities of the Gribov horizon 25

7.3.1 General idea 25

7.3.2 Degenerate perturbation theory 26

7.3.3 Two-fold degeneracy 27

7.3.4 Three-fold degeneracy 27

7.3.5 Over-all picture of the Gribov horizon and its center-vortex singularities 28

8. Coulomb gauge as an attractive fixed point of the interpolating gauge 28

9. Conclusions 29

A. Finite-volume scaling of low-lying F-P eigenvalues 31

B. Proof of convexity of FMR in SU(2) lattice gauge theory 34

G. Renormalization-group flows toward Coulomb gauge 36 


\section{Introduction}

Of the many different ideas that have been advanced to explain quark confinement, more than one may be right, or at least partially right, and these should be related in some way. As in the old story of six blind men describing an elephant, those of us concerned with the QCD confinement mechanism might benefit from unifying some of our separate impressions, to form a better image of the entire beast.

In this article we investigate the relationship between center vortices (for a review, cf. ref. [1]) and the Gribov horizon in Coulomb gauge, whose relevance to confinement has been advocated by Gribov and Zwanziger [2]. We begin with the simple fact that in a confining theory, the energy of an isolated color charge is (infrared) infinite, and this energy is a lower bound on the color-Coulomb energy [3] (defined below). This fact implies an enhancement of the density of near-zero eigenvalues $\lambda_{n}$

$$
M \phi^{(n)}=\lambda_{n} \phi^{(n)}
$$

of the Faddeev-Popov (F-P) operator in Coulomb gauge

$$
M=-\nabla \cdot \mathcal{D}(A),
$$

where $\mathcal{D}(A)$ is the covariant derivative. The F-P eigenvalue density is an observable which we are able to calculate numerically (section 2), via lattice Monte Carlo and sparse-matrix techniques. Applying standard methods [4] we are able to separate any thermalized lattice configuration into vortex-only and vortex-removed components, and we find that the enhancement of the F-P eigenvalue density can be entirely attributed to the vortex component of the gauge fields. Vortices are associated with an enhancement in the density of F-P eigenvalues $\lambda$ near $\lambda=0$; this enhancement is key to the divergence of the color-Coulomb energy in Coulomb gauge. It is absent in the Higgs phase of a gauge-Higgs system (section 3), where remnant gauge symmetry is broken [5], and vortices cease to percolate. In particular, we compare the F-P eigenvalue density found in the Higgs phase to the corresponding density for configurations in the confined phase, with vortices removed. These densities are identical in form. The density of F-P eigenvalues in the high-temperature deconfined phase of pure gauge theory is examined in section 4 . We find that the linearly rising, unscreened color-Coulomb potential, which is present in the deconfined phase [5], is associated with an enhanced density of F-P eigenvalues, and that this enhancement is again attributable to the vortex component of the gauge field. Divergence of the color-Coulomb energy is a necessary but not sufficient condition for color confinement, and this phase provides an interesting example where the infinite color-Coulomb potential gets screened. Although the horizon scenario was invented to describe confinement, it nicely accounts for the divergence of the color-Coulomb energy in the deconfined phase, as explained in the Conclusion. It is also shown (section 5) how an array of center vortices, set up "by hand" to simulate some aspects of a percolating vortex configuration, leads to an accumulation of F-P eigenvalues near $\lambda=0$.

In section 7 we demonstrate that center configurations (equivalently: thin center vortices) have some remarkable geometrical properties in lattice configuration space. First, as 
already shown in ref. [5], center configurations lie on the Gribov horizon. It is known that the Gribov horizon is a convex manifold for continuum gauge fields [6] - a result which we extend here (section [6) to lattice gauge fields - and one might therefore suspect that the Gribov horizon is also smooth and differentiable. In fact, thin vortex configurations turn out to be distinguished points on the Gribov horizon, where the manifold acquires conical or "wedge" singularities. Finally, in section 8, we point out that the Coulomb gauge condition also has a special status, in that Coulomb gauge is an attractive fixed point of a more general interpolating gauge condition, which has the Coulomb and Landau gauge conditions as special cases.

\section{The F-P eigenvalue density}

The energy of an isolated color charge is (infrared) infinite in the confined phase, even on the lattice where ultraviolet divergences are regulated. We will consider charged states in Coulomb gauge, which, for a single static point charge, has the simple form

$$
\Psi_{C}^{\alpha}[A ; x]=\psi^{\alpha}(x) \Psi_{0}[A]
$$

where $\alpha$ is the color index for a point charge in color group representation $r$, and $\Psi_{0}$ is the Coulomb gauge ground state. The gauge-field excitation energy $\mathcal{E}_{r}$ of this state, above the ground state energy, is due entirely to the non-local part of the hamiltonian

$$
\mathcal{E}_{r}=\frac{\left\langle\Psi_{C}^{\alpha}[A ; x]\left|H_{\text {coul }}\right| \Psi_{C}^{\alpha}[A ; x]\right\rangle}{\left\langle\Psi_{C}^{\alpha}[A ; x] \mid \Psi_{C}^{\alpha}[A ; x]\right\rangle}-\left\langle\Psi_{0}\left|H_{\text {coul }}\right| \Psi_{0}\right\rangle .
$$

We recall that in Coulomb gauge, the hamiltonian is a sum $H=H_{\text {glue }}+H_{\text {coul }}$, where

$$
\begin{gathered}
H_{\text {glue }}=\frac{1}{2} \int d^{3} x\left(\mathcal{J}^{-\frac{1}{2}} \vec{E}^{\mathrm{tr}, a} \mathcal{J} \cdot \vec{E}^{\mathrm{tr}, a} \mathcal{J}^{-\frac{1}{2}}+\vec{B}^{a} \cdot \vec{B}^{a}\right), \\
H_{\text {coul }}=\frac{1}{2} \int d^{3} x d^{3} y \mathcal{J}^{-\frac{1}{2}} \rho^{a}(x) \mathcal{J} K^{a b}(x, y ; A) \rho^{b}(y) \mathcal{J}^{-\frac{1}{2}},
\end{gathered}
$$

and

$$
\begin{aligned}
K^{a b}(x, y ; A) & =\left[M^{-1}\left(-\nabla^{2}\right) M^{-1}\right]_{x y}^{a b} \\
\rho^{a} & =\rho_{q}^{a}-g f^{a b c} A_{k}^{b} E_{k}^{\mathrm{tr}, c} \\
\mathcal{J} & =\operatorname{det}[-\nabla \cdot \mathcal{D}(A)] .
\end{aligned}
$$

Then the excitation energy of the charged state, in $\mathrm{SU}(N)$ gauge theory, is

$$
\mathcal{E}_{r}=g^{2} \frac{1}{d_{r}} \operatorname{Tr}\left[L^{a} L^{b}\right]\left\langle K^{a b}(x, x ; A)\right\rangle=g^{2} C_{r} \frac{1}{N^{2}-1}\left\langle K^{a a}(x, x ; A)\right\rangle,
$$

where the $\left\{L^{a}\right\}$ are color group generators, $d_{r}$ the dimension, and $C_{r}$ the quadratic Casimir of representation $r$ of the color charge. Therefore, the excitation energy $\mathcal{E}_{r}$ is the energy of the longitudinal color electric field due to the static source, which we can identify as the color Coulomb self-energy. This energy is ultraviolet divergent in the continuum, but of 
course that divergence can be regulated with a lattice cut-off. The more interesting point is that in a confining theory, $\mathcal{E}_{r}$ must still be divergent at infinite volume, even after lattice regularization, due to infrared effects.

The excitation energy (2.2) represents the (infrared-infinite) energy of unscreened color charge in the state (2.1), which in general is a highly excited state that is not an eigenstate of the hamiltonian. States of this kind are useful for extracting the self-energy of an isolated charge due to its associated color-Coulomb field. On the other hand, the minimal free energy $\mathcal{E}_{s}$ of a state containing a static external charge, at inverse temperature $T$, is obtained from the value of the Polyakov loop $P \sim \exp \left(-\mathcal{E}_{s} T\right)$. This minimal energy may be infrared finite in an unconfined phase even if $\mathcal{E}_{r}$ is not, providing the external charge can be screened by dynamical matter fields, or by high-temperature effects. The infrared divergence of $\mathcal{E}_{r}$ must be understood as a necessary, but not sufficient, condition for confinement.

We note in passing that the charged state (2.1) in Coulomb gauge corresponds, in QED in temporal gauge, to the well-known form

$$
\Psi^{Q E D}[A ; x]=\exp \left[i e \int d^{3} z A(z) \cdot \nabla \frac{1}{4 \pi|x-z|}\right] \psi(x) \Psi_{0}^{Q E D}[A]
$$

The investigation of this type of "stringless" state with external charges in non-abelian theories, using perturbative methods, was undertaken some time ago by Lavelle and McMullan in ref. [7]. The exponential prefactor in eq. (2.5) can be identified as the gauge transformation taking an arbitrary configuration $A_{k}(x)$ into Coulomb gauge. This feature generalizes to non-abelian theories, and "stringless" states with static charges in temporal gauge can be formally expressed in terms of the gauge transformation to Coulomb gauge, as shown in ref. [5].

We now proceed to the lattice formulation, with an $\mathrm{SU}(2)$ gauge group. The link variables can be expressed as

$$
U_{\mu}(x)=b_{\mu}(x)+i \vec{\sigma} \cdot \vec{a}_{\mu}(x), \quad b_{\mu}(x)^{2}+\sum_{c} a_{\mu}^{c}(x)^{2}=1
$$

and (when the lattice version of the Coulomb gauge condition $\nabla \cdot A=0$ is satisfied) the lattice Faddeev-Popov operator is

$$
\begin{aligned}
M_{x y}^{a b} & =\delta^{a b} \sum_{k=1}^{3}\left\{\delta_{x y}\left[b_{k}(x)+b_{k}(x-\hat{k})\right]-\delta_{x, y-\hat{k}} b_{k}(x)-\delta_{y, x-\hat{k}} b_{k}(y)\right\} \\
& -\epsilon^{a b c} \sum_{k=1}^{3}\left\{\delta_{x, y-\hat{k}} a_{k}^{c}(x)-\delta_{y, x-\hat{k}} a_{k}^{c}(y)\right\},
\end{aligned}
$$

where indices $x, y$ denote lattice sites at fixed time. Denote the Green's function corresponding to the F-P operator as ${ }^{1}$

$$
G_{x y}^{a b}=\left[M^{-1}\right]_{x y}^{a b}=\sum_{n} \frac{\phi_{x}^{a(n)} \phi_{y}^{b(n) *}}{\lambda_{n}},
$$

\footnotetext{
${ }^{1}$ This expression assumes $M$ is invertible, a point which will be discussed in subsection 2.1, below.
} 
where $\phi_{x}^{a(n)}, \lambda_{n}$ are the $n$-th normalized eigenstate and eigenvalue of the lattice F-P operator $M_{x y}^{a b}$. Defining the representation-independent factor $\mathcal{E} \equiv \mathcal{E}_{r} /\left(g^{2} C_{r}\right)$ in the Coulomb self-energy, we find

$$
\begin{aligned}
\mathcal{E} & =\frac{1}{N^{2}-1}\left\langle K^{a a}(x, x ; U)\right\rangle \\
& =\frac{1}{3} \sum_{y_{1} y_{2}}\left\langle G_{x y_{1}}^{a b}\left(-\nabla^{2}\right)_{y_{1} y_{2}} G_{y_{2} x}^{b a}\right\rangle=\frac{1}{3 V_{3}} \sum_{x} \sum_{y_{1} y_{2}}\left\langle G_{x y_{1}}^{a b}\left(-\nabla^{2}\right)_{y_{1} y_{2}} G_{y_{2} x}^{b a}\right\rangle \\
& =\frac{1}{3 V_{3}} \sum_{x} \sum_{y_{1} y_{2}} \sum_{m} \sum_{n}\left\langle\frac{\phi_{x}^{a(m)} \phi_{y_{1}}^{b(m) *}}{\lambda_{m}}\left(-\nabla^{2}\right)_{y_{1} y_{2}} \frac{\phi_{y_{2}}^{b(n)} \phi_{x}^{a(n) *}}{\lambda_{n}}\right\rangle \\
& =\frac{1}{3 V_{3}} \sum_{y_{1} y_{2}} \sum_{n}\left\langle\frac{\phi_{y_{1}}^{a(n) *}\left(-\nabla^{2}\right)_{y_{1} y_{2}} \phi_{y_{2}}^{a(n)}}{\lambda_{n}^{2}}\right\rangle,
\end{aligned}
$$

where $V_{3}=L^{3}$ is the lattice 3 -volume. Also defining

$$
F_{n}=\sum_{y_{1} y_{2}} \phi_{y_{1}}^{a(n) *}\left(-\nabla^{2}\right)_{y_{1} y_{2}} \phi_{y_{2}}^{a(n)}=\vec{\phi}^{(n) *} \cdot\left(-\nabla^{2}\right) \vec{\phi}^{(n)}
$$

we have

$$
\mathcal{E}=\frac{1}{3 V_{3}} \sum_{n}\left\langle\frac{F_{n}}{\lambda_{n}^{2}}\right\rangle
$$

The Faddeev-Popov operator, on the lattice, is a $3 V_{3} \times 3 V_{3}$ sparse matrix; the number of linearly-independent eigenstates is therefore $3 V_{3}$. Let $N(\lambda, \lambda+\Delta \lambda)$ be the number of eigenvalues in the range $[\lambda, \lambda+\Delta \lambda]$. We define, on a large lattice, the normalized density of eigenvalues

$$
\rho(\lambda) \equiv \frac{N(\lambda, \lambda+\Delta \lambda)}{3 V_{3} \Delta \lambda} .
$$

Then as the lattice volume tends to infinity,

$$
\mathcal{E}=\int_{0}^{\lambda_{\max }} \frac{d \lambda}{\lambda^{2}} \rho(\lambda) F(\lambda)
$$

where it is understood that the integrand is averaged over the ensemble of configurations. From this we derive a condition for the confinement phase: The excitation energy $\mathcal{E}_{r}=$ $g^{2} C_{r} \mathcal{E}$ of a static, unscreened color-charge is divergent if, at infinite volume,

$$
\lim _{\lambda \rightarrow 0} \frac{\rho(\lambda) F(\lambda)}{\lambda}>0
$$

In perturbation theory, at zero-th order in the gauge-coupling, the Faddeev-Popov operator is simply a laplacian, whose eigenstates are plane waves. Then $\lambda=k^{2}$, where $\vec{k}$ is the momentum, and from the volume element of momentum space it is easy to see that to zeroth order, in the limit of infinite lattice volume,

$$
\rho(\lambda)=\frac{\lambda^{1 / 2}}{4 \pi^{2}}, \quad F(\lambda)=\lambda,
$$


which obviously does not satisfy the confinement condition (2.14). At zeroth-order we have $\mathcal{E}=\lambda_{\text {max }}^{1 / 2} /\left(2 \pi^{2}\right)$.

We now recall briefly some aspects of the Gribov horizon scenario [2]. The lattice version of the Coulomb gauge-fixing condition $\nabla \cdot A=0$ is satisfied by any lattice configuration $U$ such that

$$
R=\sum_{x} \sum_{k=1}^{3} \operatorname{Tr}\left[g(x) U_{k}(x) g^{-1}(x+\widehat{k})\right]
$$

is stationary at the trivial gauge transformation $g=I$. The Faddeev-Popov operator is obtained from the second derivative of $R$ with respect to gauge transformations, so if $R$ is a local maximum, then all the eigenvalues of the Faddeev-Popov operator are positive. The subspace of configuration space satisfying this condition is known as the Gribov region, and it is bounded by the Gribov horizon, where $M$ develops a zero eigenvalue. In principle, the functional integral in minimal Coulomb gauge should be restricted to a subspace of the Gribov region in which the gauge fields are global maxima of $R$; this subspace is known as the "Fundamental Modular Region." Part of the boundary of the fundamental modular region lies on the Gribov horizon.

The dimension of lattice configuration space is of course very large, on the order of the number of lattice sites, and it has been proven that, in contrast to an abelian theory, the Gribov region is bounded and convex (cf. [6] and section [6 below). A suggestive analogy is that of a sphere in $N$-dimensions, which has a volume element proportional to $r^{N-1} d r$, so that most of the volume is concentrated close to the surface of the sphere. By this analogy, it is reasonable to suppose that the volume of the Gribov region is concentrated near the Gribov horizon. If that is so, then typical configurations generated by lattice Monte Carlo, fixed to Coulomb gauge by standard over-relaxation techniques, will also lie very close to the Gribov horizon, and the F-P operator for such configurations will acquire near-zero eigenvalues. This is not enough by itself to ensure confinement; even the laplacian operator will have a spectrum of near-zero modes at large lattice volumes. The conjecture is that typical configurations near the Gribov horizon may also have enhanced values for $\rho(\lambda)$ and $F(\lambda)$ (compared to the perturbative expressions) at $\lambda \rightarrow 0$, such that the confinement condition (2.14) is satisfied. Our task is to check, numerically, whether this enhancement exists or not.

\subsection{Observables}

We apply the ARPACK routine [8], which employs a variant of the Arnoldi procedure for sparse matrices, to evaluate the lowest 200 eigenvectors and corresponding eigenvalues of

the F-P matrix $M_{x y}^{a b}$ in eq. (2.7), for configurations generated by lattice Monte Carlo. The first three eigenvalues are zero for $\mathrm{SU}(2)$ gauge theory, regardless of the lattice configuration, due to the fact that the eigenvector equation (1.1) is trivially satisfied by three linearly independent, spatially constant eigenvectors

$$
\phi_{x}^{a(n)}=\frac{1}{\sqrt{V_{3}}} \delta_{a n}, \quad n=1,2,3
$$


with zero eigenvalue.

The existence of these trivial zero modes is related to the fact that physical states with non-zero total color charge cannot exist in a finite volume with periodic boundary conditions. This is true even for an abelian theory, and the reason is simple: the Gauss law cannot be satisfied for total non-zero charge in a finite periodic lattice. In such cases, the electric flux lines diverging from point sources have nowhere to end. This means that the F-P operator (or the laplacian, in an abelian theory) is non-invertible on a periodic lattice. It is precisely the existence of the trivial zero modes which makes the F-P operator non-invertible; there is no such difficulty in an infinite volume. In order to extrapolate our results on finite volumes to infinite volume, which allows non-zero total charge, there are two possibilities. First, we could get rid of zero modes by imposing non-periodic (e.g. Dirichlet) boundary conditions on the finite lattice. Second, we could perform our Monte Carlo simulations on finite periodic lattices as usual, but drop the trivial zero modes before extrapolating our results to infinite volume. In this article we choose the second approach, and exclude the trivial zero modes from all sums over eigenstates.

The average eigenvalue density $\rho(\lambda)$ is obtained from the remaining 197 eigenvalues in each thermalized configuration (there are $L$ such configurations in a given $L^{4}$ lattice, one at each time-slice). The range of eigenvalues is divided into a large number of subintervals, and eigenvalues are binned in each subinterval to determine the expected number $N(\lambda, \lambda+\Delta \lambda)$ of eigenvalues per configuration falling into each bin. Substituting this value into the definition 2.12) of the normalized density of states, we obtain an approximation to $\rho(\lambda)$ for $\lambda$ values in the middle of each subinterval. We also compute the expectation value of the $n$-th eigenvalue and corresponding quantity $F\left(\lambda_{n}\right)$

$$
\left\langle\lambda_{n}\right\rangle \text { and }\left\langle F_{n}\right\rangle \equiv\left\langle\vec{\phi}^{(n) *} \cdot\left(-\nabla^{2}\right) \vec{\phi}^{(n)}\right\rangle
$$

for the $n=4-200$ non-trivial eigenvectors. Our plots of $F(\lambda)$ vs. $\lambda$, shown below, are obtained by plotting $\left\langle F\left(\lambda_{n}\right)\right\rangle$ vs. $\left\langle\lambda_{n}\right\rangle$. Finally, we calculate the average contribution of low-lying eigenstates with $\lambda_{n}<0.15$ to the energy $\mathcal{E}$ of unscreened color charge:

$$
\epsilon=\frac{1}{3 V_{3}} \sum_{n>3}^{\lambda_{n}<0.15}\left\langle\frac{F_{n}}{\lambda_{n}^{2}}\right\rangle .
$$

For our purposes, the precise value of the upper limit in the sum is not too important. We have chosen the upper limit $\lambda=0.15$ in order that the 200 lowest eigenvalues, on each lattice volume we have studied, include the range $0<\lambda \leq 0.15$.

\subsection{Center projection and vortex removal}

Not every configuration on the Gribov horizon satisfies the confinement condition (2.14).

For example, any purely abelian configuration in a non-abelian theory lies on the Gribov horizon (after gauge transformation to Coulomb gauge [5]) and therefore has a (non-trivial) zero eigenvalue, but not all such configurations will disorder Wilson loops, or lead to an F-P eigenvalue spectrum satisfying eq. (2.14). The center vortex theory of confinement holds that a particular class of field configurations dominates the vacuum state at large 
scales, and is responsible for the linear rise of the static quark potential. If so, then these same configurations should be responsible for the pileup of F-P eigenvalues near $\lambda=0$, resulting in the infinite energy of an isolated color charge. This is the connection which we think must exist between the center vortex and Gribov horizon confinement scenarios.

To investigate this connection, we apply standard methods to factor a lattice configuration into its vortex and non-vortex content. This is done by first fixing to direct maximal center gauge, which is the Landau gauge condition in the adjoint representation

$$
R=\sum_{x} \sum_{\mu} \operatorname{Tr}\left[U_{\mu}(x)\right]^{2} \text { is maximum }
$$

using an over-relaxation technique. The lattice configuration is factored into

$$
U_{\mu}(x)=Z_{\mu}(x) \widetilde{U}_{\mu}(x)
$$

where

$$
Z_{\mu}(x)=\operatorname{sign}\left\{\operatorname{Tr}\left[U_{\mu}(x)\right]\right\}
$$

is the center-projected configuration, and $\widetilde{U}_{\mu}$ is the "vortex-removed" configuration. The center-projected (thin vortex) configuration $Z_{\mu}(x)$ carries the fluctuations which give rise to an area law for Wilson loops. The asymptotic string tension of the vortex-removed configuration $\widetilde{U}_{\mu}(x)$ vanishes, as does its chiral condensate and topological charge. The numerical evidence supporting these statements is reviewed in ref. [1].

In our procedure, each thermalized lattice configuration is transformed to direct maximal center gauge and factored as above into a center-projected configuration $Z_{\mu}(x)$, and a vortex-removed configuration $\widetilde{U}_{\mu}(x)$. These are then transformed separately into Coulomb gauge. Of course, any center-projected configuration $Z_{\mu}(x)$, with links $= \pm I$, trivially fulfills the Coulomb gauge condition

$$
\sum_{k} \operatorname{Tr}\left[\sigma^{a}\left(U_{k}(x)+U_{k}^{\dagger}(x-\widehat{k})\right)\right]=0,
$$

but in general such configurations are far from the minimal Coulomb gauge, and are normally not even in the Gribov region. So in practice we perform a random gauge transformation on $Z_{\mu}(x)$, and then fix to a gauge copy in the Gribov region by the usual over-relaxation method. We will refer to such copies as "vortex-only" configurations. Applying the Arnoldi algorithm to calculate the F-P eigenvectors and eigenvalues, we compute observables $\epsilon, \rho(\lambda),\left\langle F_{n}\right\rangle,\left\langle\lambda_{n}\right\rangle$ for both the vortex-only and vortex-removed configurations.

Any purely center configuration lies on the Gribov horizon. By "center configuration" we mean a lattice all of whose link variables can be transformed, by some gauge transformation, to center elements of the gauge group, and the "vortex-only" configurations are center configurations in this sense. It was shown in ref. [5] that for the $\mathrm{SU}(2)$ gauge group, such configurations have in general three non-trivial F-P zero modes, in addition to the three trivial, spatially constant zero modes (2.17). ${ }^{2}$ In computing $\epsilon$ for the vortex-only configurations, we therefore exclude the first six eigenvalues.

\footnotetext{
${ }^{2}$ In some special cases of high symmetry there may be fewer non-trivial zero modes.
} 


\subsection{Results}

Most of our simulations have been carried out at $\beta=2.1$. This is not such a weak coupling, but it allows us to use modestly sized lattices whose volumes are nonetheless quite large compared to the confinement scale, and to study the volume dependence. The F-P observables are calculated in the full configurations, the thin-vortex configurations, and the vortex-removed configurations, each of which has been transformed to Coulomb gauge.

Figures 11 and 2 show our results for $\rho(\lambda)$ and $F(\lambda)$ for the full configurations, on a variety of lattice volumes ranging from $8^{4}$ to $20^{4}$ (to reduce symbol overlap near $\lambda=0$, we do not display the entire set of data points in $F(\lambda)$ ). The apparent sharp "bend" in $\rho(\lambda)$ near $\lambda=0$ becomes increasingly sharp, and happens ever nearer $\lambda=0$, as the lattice volume increase. The impression these graphs convey is that in the limit of infinite volume, both $\rho(\lambda)$ and $F(\lambda)$ go to positive constants as $\lambda \rightarrow 0$. However, for both $\rho(\lambda)$ and $F(\lambda)$ we cannot exclude the possibility that the curves behave like $\lambda^{p}, \lambda^{q}$ near $\lambda=0$, with $p, q$ small powers. If we assume that the low-lying eigenvalue distribution scales with the total number of eigenvalues $\left(3 L^{3}\right)$ in the manner suggested by random matrix theory, then it is possible to deduce, from the probability distribution of the lowest non-zero eigenvalue, the power dependence of $\rho(\lambda)$ near $\lambda=0$. This analysis is carried out in Appendix $\mathbb{A}$, and gives us the estimates

$$
\rho(\lambda) \sim \lambda^{0.25}, F(\lambda) \sim \lambda^{0.38} \quad \text { full configurations }
$$

at small $\lambda$ and large volume, with perhaps a $20 \%$ error in the exponents. With this behavior the Coulomb confinement condition is satisfied, and the Coulomb self-energy is infrared divergent.

In Fig. 3 we plot $\epsilon$ vs. lattice extension $L$, together with a best straight-line fit through the points at $L=10-20$. The cut-off energy $\epsilon$ rises with $L$, and this rise is consistent with linear, although the data is not really good enough to determine the precise $L$-dependence.

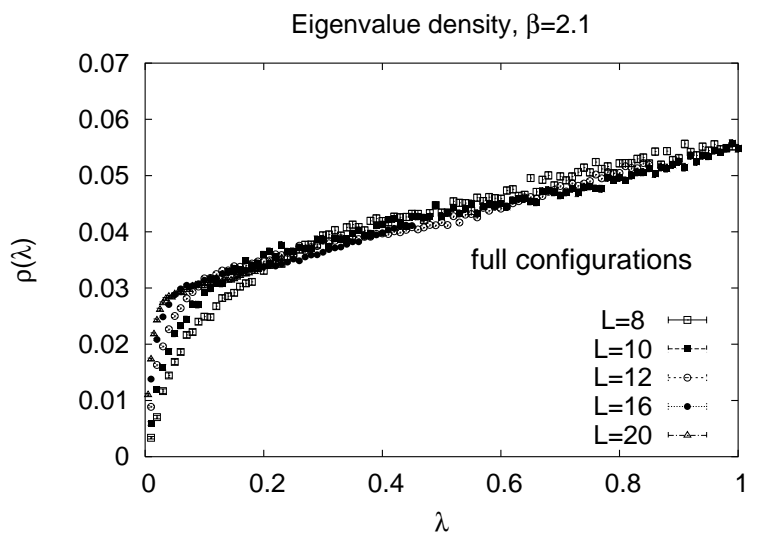

Figure 1: The F-P eigenvalue density at $\beta=2.1$, on $8^{4}-20^{4}$ lattice volumes. 


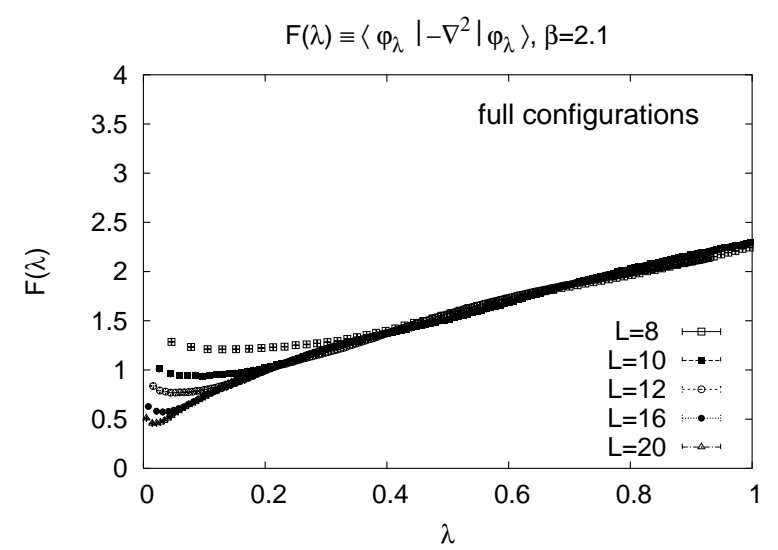

Figure 2: $F(\lambda)$, the diagonal matrix element of $\left(-\nabla^{2}\right)$ in F-P eigenstates, plotted vs. F-P eigenvalue.

One might wonder how it is possible that a pair of quark-antiquark charges, in a global color singlet combination, can have a finite total Coulomb energy in an infinite volume, given that each charge has a divergent self-energy. This question was addressed in ref. [5], which computed Coulomb energies from timelike link correlators. The answer is that both the quark self-energies, and the energy due to instantaneous one-gluon exchange between separated sources, contain an infrared divergent constant. It can be shown that for global color singlets these constants precisely cancel, while in non-singlets the self-energy is not entirely cancelled, and the total energy is infrared divergent.

Next we consider the F-P observables for the "vortex-only" configurations, consisting of thin vortex configurations (in Coulomb gauge) which were extracted from thermalized lattices as described above. Our data for $\rho(\lambda)$ and $F(\lambda)$ at the same range $\left(8^{4}-20^{4}\right)$ of

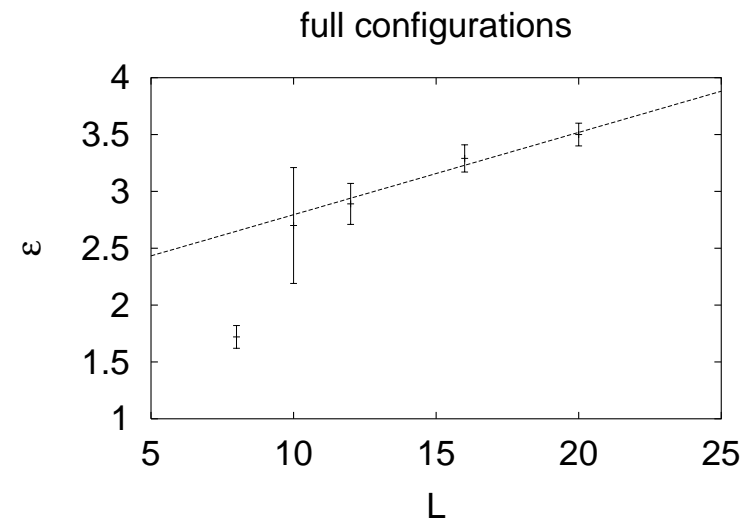

Figure 3: $\epsilon$ vs. lattice extension $L$, for full, unprojected lattice configurations. The straight line is a linear fit through points at $L=10-20$. 


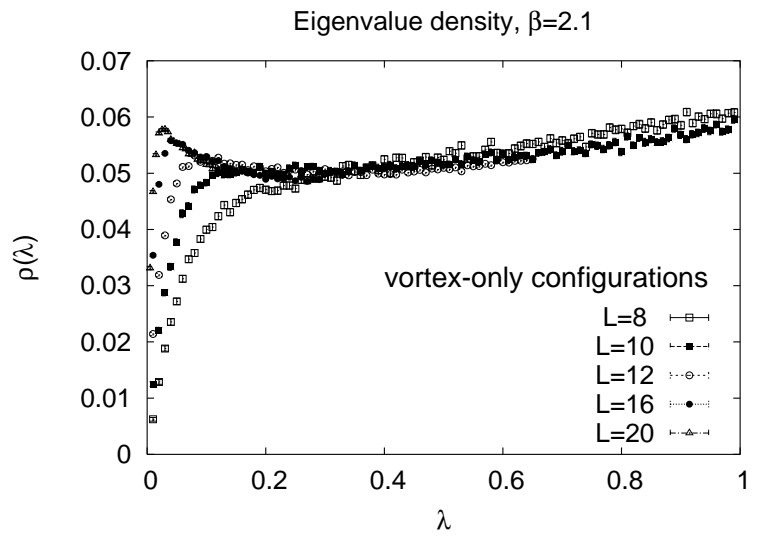

Figure 4: F-P eigenvalue density in vortex-only configurations.

lattice volumes is displayed in Figs. 4 and 5 . The same qualitative features seen for the full configurations, e.g. the sharp bend in the eigenvalue density near $\lambda=0$, becoming sharper with increasing volume, are present in the vortex-only data as well, and if anything are more pronounced. From the graphs, it would appear that

$$
\rho(0) \approx 0.06, \quad F(0) \approx 1.0, \quad \text { vortex-only }
$$

This non-zero limit for $\rho(\lambda), F(\lambda)$ at $\lambda \rightarrow 0$ is supported by an analysis of the loweigenvalue universal scaling behavior as a function of $L$, which is reported in Appendix $\mathrm{A}$. Once again, the confinement criterion (2.14) is obviously satisfied.

Figure 6 shows our data for $\epsilon(L)$, again with a linear fit through the data points at $L=10-20$, although the linear dependence is not really established.

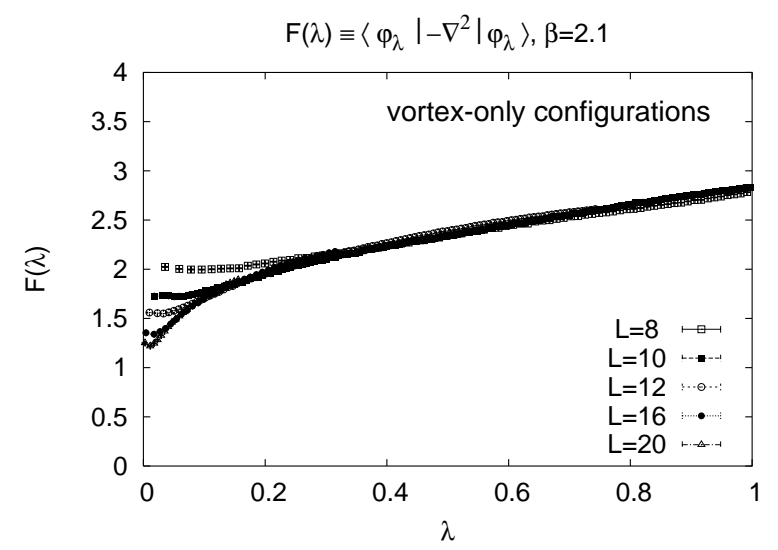

Figure 5: $F(\lambda)$, the diagonal matrix element of $\left(-\nabla^{2}\right)$ in F-P eigenstates, for vortex-only configurations. 


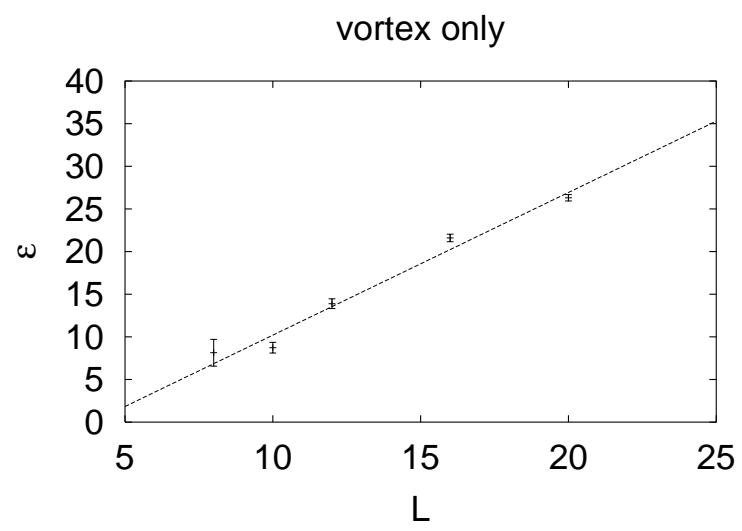

Figure 6: $\epsilon$ vs. lattice extension $L$, for the vortex-only lattice configurations. The straight line is a linear fit through points at $L=10-20$.

Finally, we consider the F-P observables of the vortex-removed configurations $\widetilde{U}$ transformed to Coulomb gauge. Our results are shown in Fig. 团 for $\rho(\lambda)$, and Fig. 8 for $F(\lambda)$. The behavior of these observables is strikingly different, in the vortex-removed configurations, from what is seen in the full and vortex-only configurations. A graph of the eigenvalue density, at each lattice volume, shows a set of distinct peaks, while the data for $F(\lambda)$ is organized into bands, with a slight gap between each band. Closer inspection shows that eigenvalue interval associated with each band in $F(\lambda)$ precisely matches the eigenvalue interval of one of the peaks in $\rho(\lambda)$.

In order to understand these features, we consider the eigenvalue density of the F-P operator $M_{x y}^{a b}=$\begin{tabular}{ccccc}
$k$ & $N_{k}$ & $n_{i}$ & $\lambda_{k}$ \\
\hline & 3 & $(0,0,0)$ & 0
\end{tabular} $\delta^{a b}\left(-\nabla^{2}\right)_{x y}$ appropriate to an abelian theory (or a $\quad \begin{array}{ccccc} & & 18 & (1,0,0) & 4 \sin ^{2}(\pi / L)\end{array}$ non-abelian theory at zero-th order in the coupling). $\quad 3 \quad 36 \quad(1,1,0) \quad 8 \sin ^{2}(\pi / L)$ $\begin{array}{lllll}\text { Although we can readily derive the result } \rho(\lambda) \sim \sqrt{\lambda} & 3 & 36 & (1,1,0) & 8 \sin ^{2}(\pi / L) \\ 4 & 24 & (1,1,1) & 12 \sin ^{2}(\pi / L)\end{array}$ (2.15) at infinite volume, this result is slightly mis- \begin{tabular}{rrrr}
5 & 18 & $(2,0,0)$ & $4 \sin ^{2}(2 \pi / L)$ \\
\hline
\end{tabular} leading at finite volume, where the eigenvalue density is actually a sum of delta-functions

$$
\rho(\lambda)=\frac{1}{3 V_{3}} \sum_{k} N_{k} \delta\left(\lambda-\lambda_{k}\right)
$$

Table 1: Eigenvalues $\lambda_{k}$ of the zerofield lattice F-P operator $-\delta^{a b} \nabla^{2}$, and their degeneracies $N_{k}$.

In the sum, the index $k$ labels the distinct eigenvalues of the lattice laplacian $-\nabla^{2}$, and $N_{k}$ is the degeneracy of $\lambda_{k}$. Explicitly, to each distinct eigenvalue $\lambda_{k}$ on an $L^{4}$ lattice, there is a set of integers $n_{1-3}$ such that

$$
\lambda_{k}=4 \sum_{i=1}^{3} \sin ^{2}\left[\frac{\pi n_{i}}{L}\right] .
$$

The first few values of $\lambda_{k}$, and their degeneracies, are listed in Table 1. 


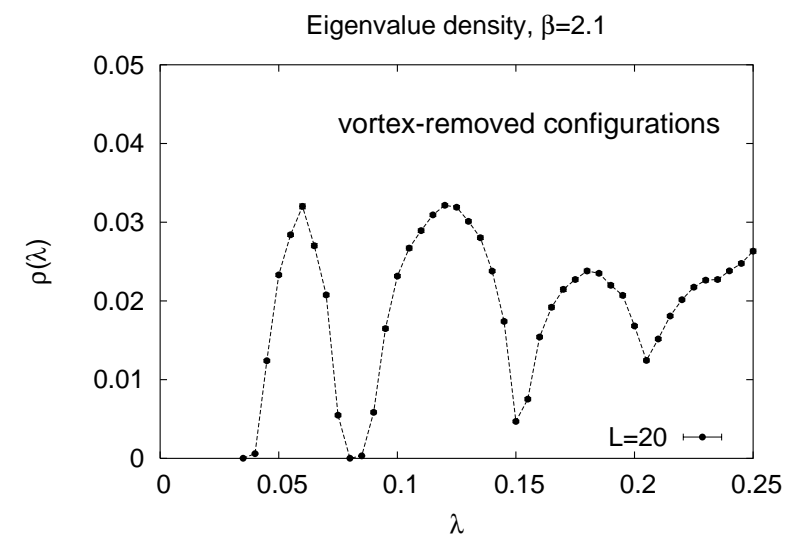

Figure 7: F-P eigenvalue densities for vortex-removed configurations, on a $20^{4}$ lattice volume.

We have compared these degeneracies $N_{k}$, of the zeroth-order F-P eigenvalues, with the number of eigenvalues per lattice configuration found inside the $k$-th "peak" of $\rho(\lambda)$, and $k$ th "band" of $F(\lambda)$. We find there is a precise match. This leads to a simple interpretation: the vortex-removed configuration $\widetilde{U}_{\mu}$ can be treated as a small perturbation of the zero-field limit $U_{\mu}=I$. This perturbation lifts the degeneracy of the $\lambda_{k}$, spreading the degenerate eigenvalues into the bands of finite width in $\lambda$. At least for small $k$, these bands do not overlap. Likewise, the perturbation broadens the infinitely narrow $\delta$-function peaks in the density of eigenstates, eq. (2.26), into the peaks of finite width seen in Fig. 0 .

Because both the density of eigenvalues and the data for $F(\lambda)$ seem to be only a perturbation of the corresponding zero-field results, it appears to be most unlikely that the

$$
F(\lambda) \equiv\left\langle\varphi_{\lambda}\left|-\nabla^{2}\right| \varphi_{\lambda}\right\rangle \text {, vortex-removed configurations }
$$
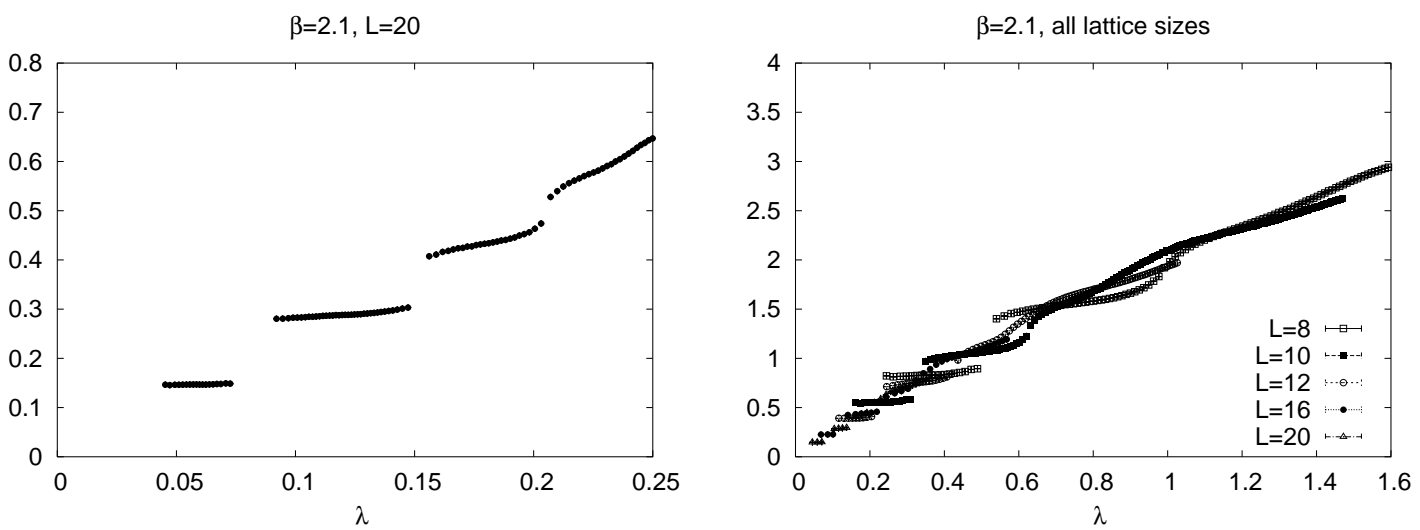

Figure 8: $F(\lambda)$ vs. $\lambda$ in the vortex-removed configurations. We display results for the $20^{4}$ volume alone (left), and a variety of lattice volumes (right). 
no-vortex configurations lead to a divergent Coulomb self-energy. In fact, we find that the low-lying eigenvalue spectrum scales with lattice extension $L$ as

$$
\left\langle\lambda_{n}\right\rangle \sim \frac{1}{L^{2}}
$$

just as in the zero-field (or zero-th order) case. We have not plotted $\epsilon(L)$ for the vortex removed case, because for the smallest lattice volume no eigenvalues actually lie in the given range $0<\lambda<\lambda_{\min }$, and even at $L=10,12$ only a few of the lowest eigenvalues are in this range. We do note, however, that the values of $\epsilon$ on the large lattices are roughly two orders of magnitude smaller than the corresponding values for the full configurations.

Vortex removal is in some sense a minimal disturbance of the original lattice configuration. The procedure only changes field strengths at the location of P-vortex plaquettes, and the fraction of $\mathrm{P}$-plaquettes out of the total number of plaquettes on the lattice dives exponentially to zero with increasing $\beta$. Nevertheless, this modest change of the lattice configuration is known to set the string tension and topological charge to zero, and to remove chiral symmetry breaking. At $\beta=2.1$, we have found that vortex removal also drastically affects the density of low-lying eigenvalues, and one sees, in the multi-peak structure of $\rho(\lambda)$, the remnants of the delta-function peaks of the free theory. We have used this comparatively low value of $\beta$ so that we may probe large physical distances, as compared to the distance scale set by the string tension, on rather modestly sized (up to $20^{4}$ ) lattices. This allows us carry out the finite volume scaling analysis reported in Appendix A.

However, using such a small $\beta$ has a price, in terms of our confidence in the effects of vortex removal. At $\beta=2.1$ roughly $17 \%$ of all plaquettes are P-vortex plaquettes (cf. Fig. 20 in ref. [1]), and one may object that in this case vortex removal is not such a small disturbance of the lattice configuration. Perhaps the drastic effect of vortex removal on the eigenvalue density is simply an artifact of the substantial number of plaquettes modified.

In order to address this concern, we have computed $\rho(\lambda)$ and $F(\lambda)$ at $\beta=2.3$ and $\beta=2.4$, where the $\mathrm{P}$-vortex densities have dropped to around $9 \%$ and $4 \%$, respectively, of the total number of plaquettes. The results are shown in Fig. 9, where we display the data for the unmodified, vortex-only, and vortex-removed configurations on the same plot. Only two lattice volumes are shown at each coupling. The effect of vortex removal is seen to be much the same at these higher $\beta$ values as at $\beta=2.1$. Again we see a multi-peak structure in $\rho(\lambda)$, and a band structure in $F(\lambda)$ at the low-lying eigenvalues (although the gap between bands narrows as $\beta$ increases). In each configuration, we have checked that the number of eigenvalues in each peak of $\rho(\lambda)$ matches the number of eigenvalues in each band of $F(\lambda)$, and that this number is again equal to the degeneracy of eigenvalues. Therefore, the interpretation proposed for the no-vortex data at $\beta=2.1$ appears to apply equally well at the higher $\beta$ values: these data are simply perturbations of the zero-field result, for which the eigenvalue density is a sum of delta-functions. The data for the vortex-only and unmodified configurations are also qualitatively similar to the results we have obtained at the lower $\beta=2.1$ value, although the lattice volumes, in physical units, are considerably smaller than the corresponding lattice volumes at $\beta=2.1$. 


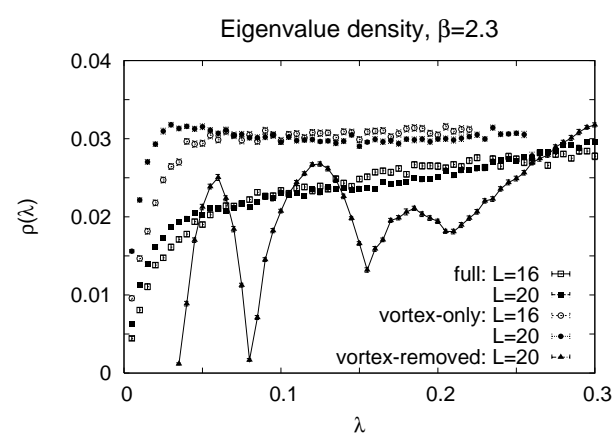

(a) $\rho(\lambda), \beta=2.3$

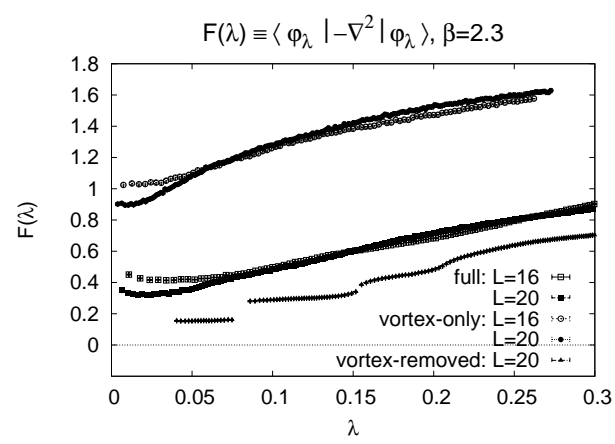

(c) $F(\lambda), \beta=2.3$

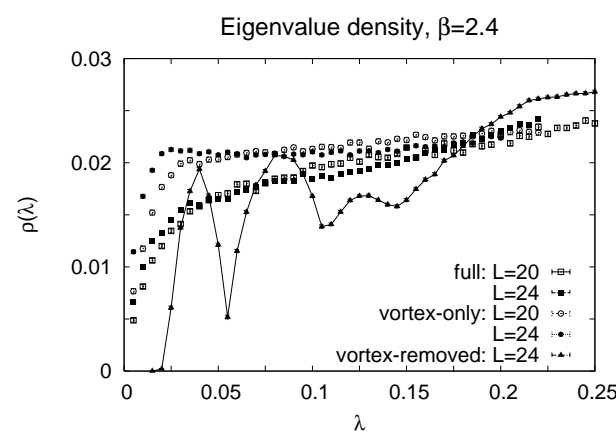

(b) $\rho(\lambda), \beta=2.4$

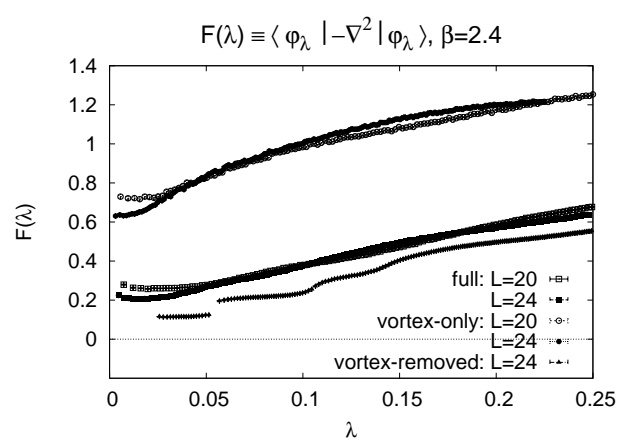

(d) $F(\lambda), \beta=2.4$

Figure 9: $\rho(\lambda), F(\lambda)$ at couplings $\beta=2.3,2.4$ for full, vortex-only, and vortex-removed configurations.

We conclude that it is the vortex content of the thermalized configurations which is responsible for the enhancement of both the eigenvalue density and $F(\lambda)$ near $\lambda=0$, leading to an infrared-divergent Coulomb self-energy.

\section{Gauge-Higgs theory}

Next we consider a theory with the gauge field coupled to a scalar field of unit modulus in the fundamental representation of the gauge group. For the $\mathrm{SU}(2)$ gauge group, the lattice action can be written in the form [9]

$$
S=\beta \sum_{\text {plaq }} \frac{1}{2} \operatorname{Tr}\left[U U U^{\dagger} U^{\dagger}\right]+\gamma \sum_{x, \mu} \frac{1}{2} \operatorname{Tr}\left[\phi^{\dagger}(x) U_{\mu}(x) \phi(x+\widehat{\mu})\right]
$$

with $\phi$ an $\mathrm{SU}(2)$ group-valued field. Strictly speaking, this theory is non-confining for all values of $\beta, \gamma$. In particular, there is no thermodynamic phase transition (non-analyticity in the free energy), from the Higgs phase to a confinement phase; this is the content of a well-known theorem by Osterwalder and Seiler [10], and Fradkin and Shenker [11]. 
However, the Osterwalder-Seiler-Fradkin-Shenker (OS-FS) theorem is not the last word on phase structure in the gauge-Higgs system. In fact, there $i s$ a symmetry-breaking transition in this theory. If one fixes to Coulomb gauge, there is still a remaining freedom to perform gauge transformations which depend only on the time coordinate. It is therefore possible, in an infinite volume, that this symmetry is broken on any given time-slice (where the remnant symmetry is global). The order parameter for the symmetry-breaking transition is the modulus of the timelike links, averaged over any time slice

$$
\begin{aligned}
& Q=\left\langle\sqrt{\operatorname{Tr}\left[\frac{1}{2} V(t) V^{\dagger}(t)\right]}\right\rangle, \\
& V=\frac{1}{L^{3}} \sum_{x} U_{0}(x, t) .
\end{aligned}
$$

If $Q \rightarrow 0$ as $L \rightarrow \infty$, then the remnant gauge symmetry is unbroken, the Coulomb potential (as opposed to the static potential) between quark-antiquark sources rises linearly, and the energy of an isolated color-charge state of form (2.1) is infrared divergent. Conversely, if $Q>0$ at infinite volume, then the remnant symmetry is broken, the Coulomb potential is asymptotically flat, and the energy of an isolated color-charge state is finite.

These matters are explained in some detail in ref. [5], where we report on a sharp transition between a symmetric ("confinement-like") phase and a broken ("Higgs") phase of remnant gauge symmetry. There is no inconsistency with the OS-FS theorem, which assures analyticity of local, gauge-invariant order parameters. The order parameter $Q$, when expressed as a gauge-invariant observable, is highly non-local. The transition line between the symmetric and broken phases is also not the location of a thermodynamic transition, in the sense of locating non-analyticity in the free energy. Rather, it is most likely a Kertész line, of the sort found in the Ising model at finite external magnetic field, which identifies a percolation transition [12]. In the gauge-Higgs case, the objects which percolate in the confinement-like phase, and cease to percolate in the Higgs phase, turn out to be center vortices [13] (see also [14, 15]).

In the previous section we investigated the effect, on F-P observables, of removing center vortices from lattice configurations by hand. The gauge-Higgs system gives us the opportunity of suppressing the percolation of vortices by simply adjusting the coupling constants; we can then study the F-P observables in screened phases with and without percolation. We note that the "confinement-like" phase is a screened phase, rather than a true confinement phase, in that the energy of a static color charge is not infinite, because it can be screened by the dynamical Higgs particles. Nevertheless, in this phase the Coulomb potential is confining, and the Coulomb energy of an isolated charged state of the form (2.1) (an unscreened charge) is infrared infinite [5]. ${ }^{3}$

In Figs. 10 and 11 we display the results for $\rho(\lambda)$ and $F(\lambda)$ on a $12^{4}$ lattice at $\beta=2.1$ and $\gamma=0.6$, which is inside the symmetric (or "confinement-like") phase. Data extracted from the full lattice, vortex-only, and vortex-removed configurations are shown on each

\footnotetext{
${ }^{3}$ The Coulomb potential is only an upper bound on the static quark potential [3]; a confining Coulomb potential is a necessary but not sufficient condition for confinement.
} 


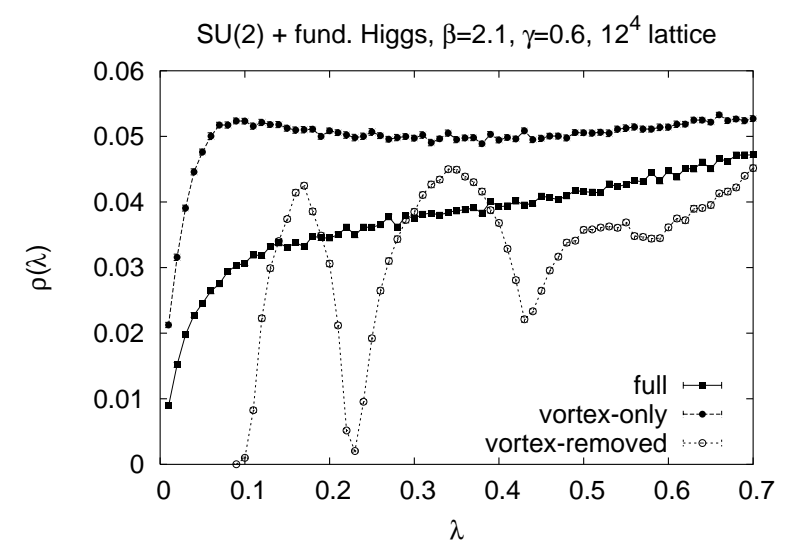

Figure 10: Eigenvalue densities for a gauge-Higgs system in the "confined", unbroken remnant symmetry phase. Data for the full, vortex-only, and vortex-removed configurations are shown, taken on a $12^{4}$ lattice.

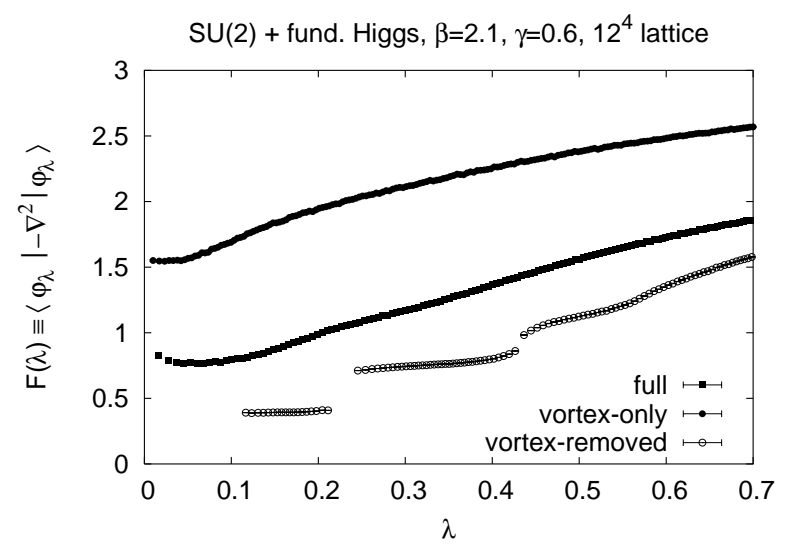

Figure 11: $F(\lambda)$ for a gauge-Higgs system in the "confined" phase, on a $12^{4}$ lattice. Data is shown for the full, vortex-only, and vortex-removed configurations.

graph, and these are qualitatively very similar to what we have already seen in the puregauge theory (equivalent to $\gamma=0$ ). But things change drastically as we move across the transition into the Higgs phase. The results for $\rho(\lambda)$ and $F(\lambda)$ at $\beta=2.1$ and $\gamma=1.2$, which is inside the Higgs phase, are shown in Figs. 12 and 13. Data, taken on a $12^{4}$ lattice, is displayed for the full configurations only. Note that these figures look almost identical to the corresponding vortex-removed data in the symmetric phase, Figs. 10 and 11, which in turn are very close to the vortex-removed data obtained in the pure gauge theory at $\beta=2.1$, on a $12^{4}$ lattice.

This last feature is worth stressing. From the point of view of the F-P operator, a thermalized configuration in a pure-gauge theory factors into a piece which actually does the confining (the vortex-only configuration), and a piece which closely resembles the lattice 


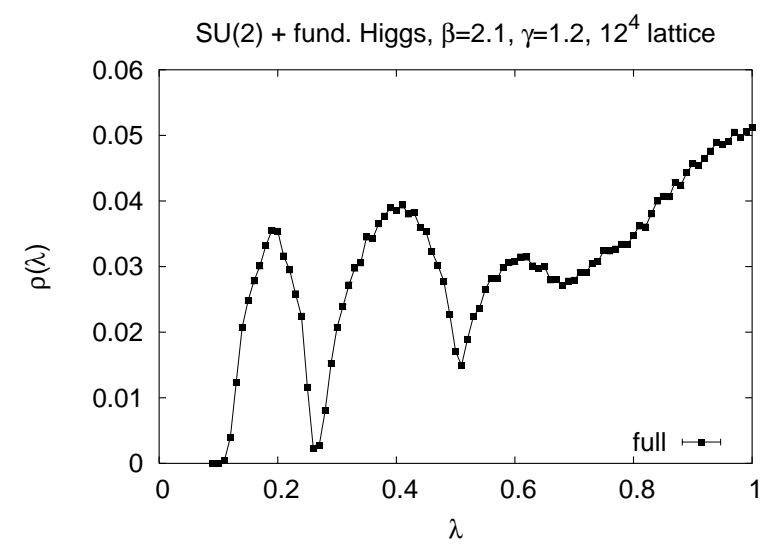

Figure 12: The F-P eigenvalue density in the Higgs (broken remnant symmetry) phase, for the full configurations.

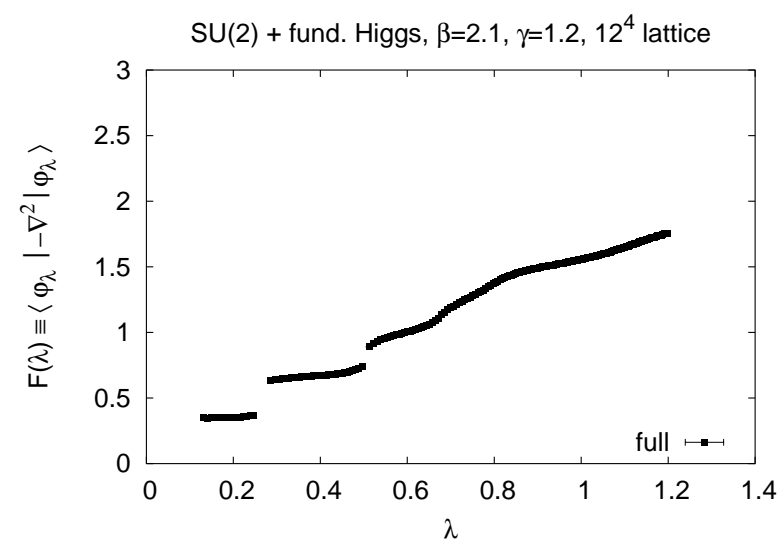

Figure 13: $F(\lambda)$ in the Higgs phase, for full configurations.

of a gauge theory in the Higgs phase (the vortex-removed configuration).

Vortex-removal in the Higgs phase does not affect the data for $\rho(\lambda)$ appreciably. ${ }^{4}$ It seems safe to conclude that this behavior of the F-P observables, found in the Higgs phase, is consistent with an infrared finite Coulomb self-energy.

\section{Eigenvalue density in the deconfined phase}

It was reported in ref. [5] that the instantaneous color Coulomb potential $V_{\text {coul }}(r)$ is linearly rising at large separation, i.e. $V_{\text {coul }}(r) \sim \sigma_{\text {coul }} r$ where $\sigma_{\text {coul }}$ is a Coulomb string tension, in the high-temperature deconfined phase of pure gauge theory. This fact is surprising at first sight, because the free energy $V(r)$ of a static quark pair in the deconfined phase

\footnotetext{
${ }^{4}$ The eigenvalue density for vortex-only configurations shows a set of sharp, narrow peaks, very much like the sum of delta-functions in the zero-field limit.
} 
is not confining. But it is not paradoxical, because $V_{\text {coul }}(r)$ is the energy of unscreened charges, and provides only an upper bound on $V(r)$ [3]. Confining behavior in $V_{\text {coul }}(R)$ is a necessary but not sufficient condition for confinement, as we have already noted.

In fact, the confining behavior of $V_{\text {coul }}(R)$ in the deconfinement phase was to be expected. The color Coulomb potential is derived from the non-local term $H_{\text {coul }}$ in the Coulomb gauge Hamiltonian, this term depends on the Faddeev-Popov operator via the expression $M^{-1}\left(-\nabla^{2}\right) M^{-1}$, and the F-P operator, on the lattice, depends only on spacelike links at a fixed time. But we know that even at very high temperatures, spacelike links at any fixed time form a confining $D=3$ dimensional lattice, and spacelike Wilson loops have an area-law falloff just as in the zero-temperature case [16. Since $V_{\text {coul }}(R)$ depends only on the three-dimensional lattice, it is natural that $V_{\text {coul }}(R)$ confines at any temperature, and that remnant gauge symmetry (as opposed to center symmetry) is realized in the unbroken phase.

The role of center vortices in producing an area law for spacelike loops at high temperatures has been discussed in refs. [17]. If the confinement property of spacelike links is eliminated by vortex removal, then by the previous reasoning we would expect $\sigma_{\text {coul }}$ to vanish as a consequence. This consequence was also verified in ref. [5].

Given these results, it may be expected that the color-Coulomb self-energy $\mathcal{E}_{r}$ of an isolated static charge is infrared divergent in the deconfined phase, and that this is associated with an enhancement of the eigenvalue density $\rho(\lambda)$ of the Faddeev-Popov operator, and of $F(\lambda)$, the expectation value of $\left(-\nabla^{2}\right)$ in F-P eigenstates.

To test this we have evaluated $\rho(\lambda)$ and $F(\lambda)$ at $\beta=2.3$ on $16^{3} \times 2$ and $20^{3} \times 2$ lattices, which is inside the deconfined phase. We have done this for the full configurations, and also for the vortex-only and vortex-removed configurations, defined as in the zero-temperature case. The results are shown in Figs. 14 and 15. The striking feature of these figures is their strong resemblance to the corresponding figures in the confined phase at zero temperature, namely Figs. 1 and 2 for full configurations, Figs. 国 and for vortex-only configurations, and Figs. 7 and 8 for the vortex-removed configurations. Although we have not attempted to determine the critical exponents in the deconfined phase, it is clear that there is an enhanced density of low-lying eigenvalues of the Faddeev-Popov operator, and that this is associated with the center-vortex content of the configuration. It is hard to avoid the conclusion that the Gribov scenario and the vortex-dominance theory apply in both the confined and deconfined phases.

\section{Thin vortices and the eigenvalue density}

In the preceding sections we have studied the eigenvalue distribution in thin vortex configurations, extracted via center projection from thermalized lattices. It is also of interest to study the eigenvalue spectrum of thin vortex configurations of some definite geometry, such as an array.

A single thin vortex, occupying two parallel planes in the four-dimensional lattice, can be constructed in the following way: Begin with the zero-field configuration, i.e. all links 


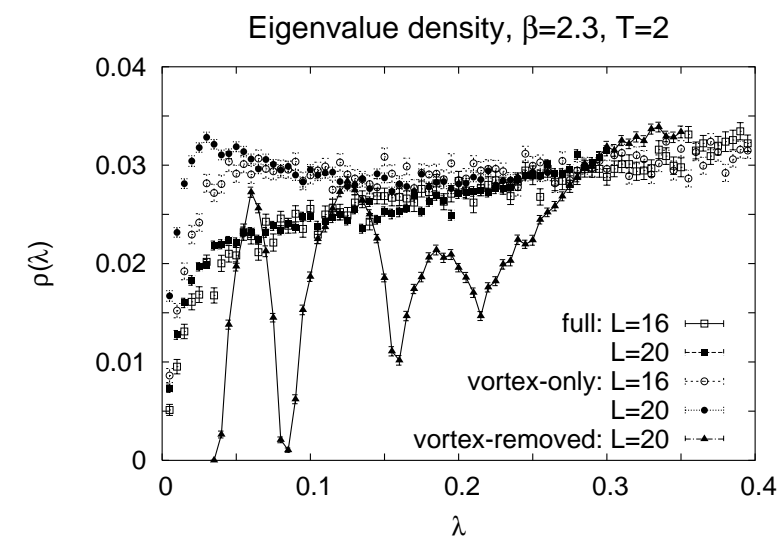

Figure 14: The F-P eigenvalue density in the deconfined phase for full, vortex-only, and vortex-removed configurations.

equal to the identity matrix. Then set $U_{2}(x, y, z, t)=-I$ at sites

$$
1 \leq x \leq \frac{L}{2}, \quad y=1, \quad \text { all } z, t .
$$

This creates two P-plaquettes in every $x y$ plane, which extends to two vortex lines (stacks of P-plaquettes along a line on the dual lattice) at a fixed time, and two vortex planes when extended also in the $t$-direction of the lattice. The two planes bound a connected (Dirac) 3-volume, and will therefore be referred to as a single closed vortex.

Generalizing slightly, we create $N$ vortices parallel to the $z t$ plane by setting

$$
U_{2}\left(x, y_{n}, z, t\right)=-I
$$

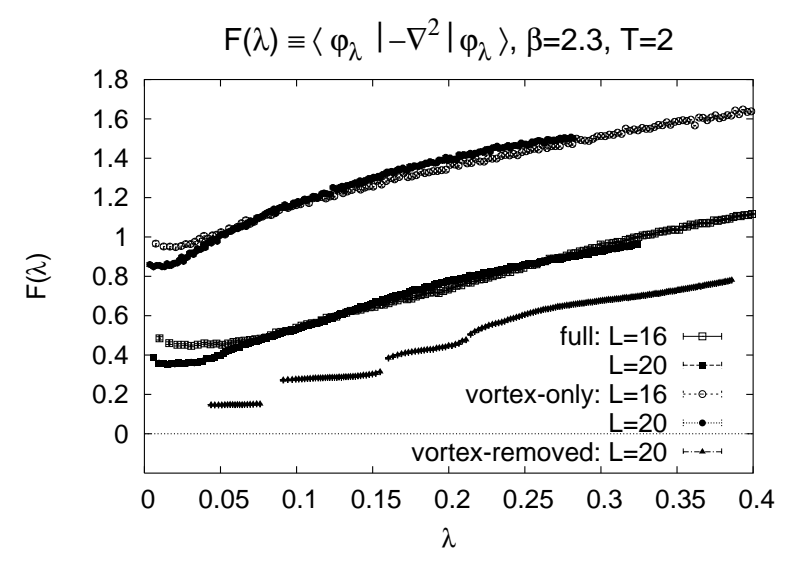

Figure 15: $F(\lambda)$ in the deconfined phase for full, vortex-only, and vortex-removed configurations. 


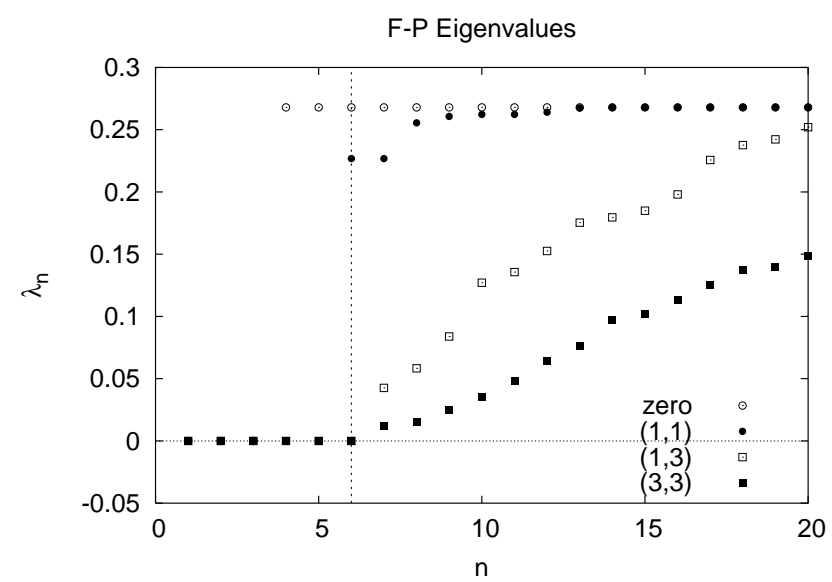

Figure 16: Distribution of low-lying eigenvalues in zero-field $(0,0)$, one-vortex $(1,1)$, and vortex array $(1,3),(3,3)$ configurations.

for

$$
1 \leq x \leq \frac{L}{2}, \quad\left\{y_{n}=1+\frac{n}{N} L, n=0,1, \ldots, N-1\right\} .
$$

In the same way (and making a few arbitrary choices of location), we create $N$ vortices parallel to the $x t$ plane by setting

$$
U_{3}\left(x, y, z_{n}, t\right)=-I
$$

for

$$
3 \leq y \leq \frac{L}{2}+2, \quad\left\{z_{n}=1+\frac{n}{N} L, n=0,1, \ldots, N-1\right\}
$$

and parallel to $y t$ plane by

$$
U_{1}\left(x_{n}, y, z, t\right)=-I
$$

for

$$
\frac{L}{2} \leq z \leq L-1, \quad\left\{x_{n}=1+\frac{n}{N} L, n=0,1, \ldots, N-1\right\} .
$$

We will consider a class of configurations characterized by the pair of integers $(N, P)$, where $P$ is the number of planar orientations (i.e. $P=1$ means only vortices parallel to the $z t$ plane, while $P=3$ means vortices parallel to the $x t$, yt and $z t$ planes), and $N$ is the number of closed vortices in each planar orientation.

As already noted, any configuration consisting of links equal to $\pm I$ trivially fulfills the Coulomb gauge condition, but also generally lies outside the Gribov region. We therefore perform a random gauge transformation on each thin vortex configuration, and fix to a Coulomb gauge copy in the Gribov region by over-relaxation. Then the F-P eigenvalue spectrum (the same at any time-slice) is extracted by the Arnoldi algorithm.

The results are shown in Fig. 16 for the first 20 F-P eigenvalues obtained on a $12^{4}$ lattice for zero-field $(0,0)$, one vortex $(1,1)$, three vortex $(1,3)$, and nine vortex $(3,3)$ configurations. As mentioned previously, it can be shown analytically that arrays of thin vortices can 
have some additional zero modes, beyond the three trivial zero modes which exist for any lattice configuration. These additional modes are seen numerically in our calculation for the vortex spectrum. Apart from these extra zero modes, we see that the low-lying eigenvalue spectrum of the one-vortex configuration is not much different from the zero-field result. But the magnitudes of the low-lying eigenvalues changes drop abruptly, compared to the laplacian $(0,0)$ eigenvalues, upon going to a three vortex configuration, and drop still further in a nine vortex array. This is only a qualitative result, but it does illustrate quite clearly the connection between vortices and the Gribov horizon. When the vortex geometry is chosen to imitate various features of percolating vortices, e.g. piercing planes in all directions and distributed throughout the lattice, then the low-lying eigenvalues have very small magnitudes as compared to the zero-field (or, in perturbation theory, zero-th order) result. This implies a pileup of F-P eigenvalues near $\lambda=0$, which we know is required for confinement.

\section{Convexity of FMR and Gribov regions in $\mathrm{SU}(2)$ lattice gauge theory}

In the section that follows this one, we will show that vortex configurations play a special role in the geometry of the fundamental modular region $\Lambda$ and of the Gribov region $\Omega$. But first we interrupt our narrative to establish a very general convexity property of these regions in lattice gauge theory.

We start by recalling the well known convexity of $\Lambda$ and $\Omega$ in continuum gauge theory [6]. The Gribov region $\Omega$ is the set of configurations that are transverse $\nabla \cdot A=0$, and for which the Faddeev-Popov operator $M(A)=-\nabla \cdot \mathcal{D}(A)$ is positive. It is also the set of all relative minima on all gauge orbits of the functional $F_{A}(g)=\left\|{ }^{g} A\right\|^{2}$, where $\|A\|$ is the Hilbert norm of the configuration $A$, and ${ }^{g} A$ is its gauge transform by $g$. The fundamental modular region $\Lambda$ is the subset of the Gribov region, $\Lambda \subset \Omega$, which consists of all absolute minima $F_{A}(1) \leq\left\|{ }^{g} A\right\|^{2}$ for all local gauge transformations $g(x)$. It is well known that in continuum gauge theory both of these regions are convex namely, if $A_{1}$ and $A_{2}$ are configurations in $\Lambda$ (or $\Omega$ ), then so is $A=\alpha A_{1}+\beta A_{2}$, where $0<\alpha<1$, and $\beta=1-\alpha$. Geometrically, $A$ lies on the line segment that joins $A_{1}$ and $A_{2}$.

We shall establish a similar, but slightly weaker property that holds in $\mathrm{SU}(2)$ lattice gauge theory. This is quite surprising because convexity is a linear concept, whereas lattice configurations are elements of a non-linear space. We parametrize $\mathrm{SU}(2)$ configurations by $U_{i}(x)=b_{i}(x)+i \vec{\sigma} \cdot \vec{a}_{i}(x)$, where $b_{i}(x)= \pm\left[1-\vec{a}_{i}^{2}(x)\right]^{1 / 2}$. The $\vec{a}_{i}(x)$ would be a complete set of coordinates, but for the sign ambiguity of $b_{i}(x)$ above, corresponding to northern and southern hemisphere. We call $\mathcal{U}_{+}$the set of configurations $U$ where all link variables lie on the northern hemisphere

$$
\mathcal{U}_{+} \equiv\left\{U: b_{i}(x)=+\left[1-\vec{a}_{i}^{2}(x)\right]^{1 / 2}\right\}
$$

for all $x$ and $i$. The $\vec{a}_{i}(x)$ are a complete set of coordinates in $\mathcal{U}_{+}$. 
In minimal Coulomb gauge the fundamental modular region $\Lambda$ has the defining property that a configuration $U$ in $\Lambda$ satisfies

$$
\sum_{x i} \operatorname{Re} \operatorname{Tr}\left[g(x)^{-1} U_{i}(x) g(x+\hat{\imath})\right] \leq \sum_{x i} \operatorname{Re} \operatorname{Tr} U_{i}(x)
$$

for all $g(x)$. Thus the gauge choice makes all the $U_{i}(x)$ as close to the identity as possible, in an equitable way over the whole lattice. In this gauge, the link variables $U_{i}(x)$ for equilibrated configurations lie overwhelmingly in the northern hemisphere, especially in the continuum limit $\beta \rightarrow \infty$. We define $\Lambda_{+}$to be the restriction of $\Lambda$ to $\mathcal{U}_{+}$, the set of configurations whose link variables all lie in the northern hemisphere

$$
\Lambda_{+} \equiv \Lambda \cap \mathcal{U}_{+}
$$

and we call it the restricted fundamental modular region. We expect that these are the important configurations in the continuum limit. This is in fact necessary if the gauge-fixed lattice theory possesses a continuum limit. In this case $\vec{a}_{i}(x) \rightarrow-2 a \vec{A}_{i}(x)$, where $a$ is the lattice spacing, and $\vec{A}_{i}(x)$ is the continuum gauge connection.

In the minimal lattice Coulomb gauge, the $\vec{a}_{i}(x)$ satisfy the lattice transversality condition

$$
[\operatorname{div} a]^{b}(x) \equiv \sum_{i}\left[a_{i}^{b}(x)-a_{i}^{b}(x-\hat{\imath})\right]=0 .
$$

This is a linear condition on the coordinates $a_{i}^{b}(x)$. This suggests that we identify configurations in $\mathcal{U}_{+}$with the space of coordinates $a_{i}^{b}(x)$, and that we add configurations by adding coordinates

$$
\vec{a}_{i}(x)=\left[\alpha \vec{a}_{1}+\beta \vec{a}_{2}\right]_{i}(x)=\alpha \vec{a}_{1, i}(x)+\beta \vec{a}_{2, i}(x) .
$$

This yields a well-defined configuration $U \in \mathcal{U}_{+}$only if $\vec{a}_{i}^{2}(x) \leq 1$ for all links. This is assured for the case that is of interest to us where $0<\alpha<1$ and $\beta=1-\alpha$. Indeed, by the triangle inequality we have

$$
\left|\vec{a}_{i}(x)\right| \leq\left|\alpha \vec{a}_{1, i}(x)\right|+\left|\beta \vec{a}_{2, i}(x)\right| \leq \alpha+\beta=1 .
$$

Statement: In $\mathrm{SU}(2)$ lattice gauge theory the restricted fundamental modular region is convex. More precisely if $a_{1}$ and $a_{2}$ lie in $\Lambda_{+}$, then $a \equiv \alpha a_{1}+\beta a_{2}$ also lies in $\Lambda_{+}$for $0<\alpha<1$ and $\beta=1-\alpha$.

The proof is given in Appendix B.

We may establish the same convexity property for the Gribov region $\Omega$, namely that $\Omega_{+} \equiv \Omega \cap \mathcal{U}_{+}$is convex. The Gribov region has the defining property

$$
\begin{aligned}
& \sum_{x i}\left\{b_{i}(x)\left[\vec{\omega}_{i}(x+\hat{\imath})-\vec{\omega}_{i}(x)\right]^{2}\right. \\
& \left.-\left[\vec{\omega}_{i}(x+\hat{\imath})-\vec{\omega}_{i}(x)\right] \cdot \vec{a}_{i}(x) \times\left[\vec{\omega}_{i}(x+\hat{\imath})+\vec{\omega}_{i}(x)\right]\right\} \geq 0,
\end{aligned}
$$

for all $\omega$. The proof for $\Omega$ is the same as for $\Lambda$ because this inequality has the same structure as (6.2), being linear in $a_{i}(x)$ and $b_{i}(x)$. 


\section{Vortices as vertices}

We have seen that $\Omega_{+}$is convex, so one might think it has a simple oval shape. We shall show however that when thin center vortex configurations are gauge transformed into minimal Coulomb gauge they are mapped into points $U_{0}$ on the boundary $\partial \Omega$ of the Gribov region $\Omega$ where this boundary has a wedge-conical singularity of a type that is described below. This illustrates the intimate relation of dominance by configurations in the neighborhood of the Gribov horizon or in the neighborhood of thin center vortex configurations.

\subsection{Previous results}

We call a "thin center vortex configuration" (or center configuration) one where, in maximal center gauge, all link variables are center elements $U_{i}(x)=Z_{i}(x)$. [Such a configuration may be characterized in a gauge-invariant way by the statement that all holonomies are center elements. (A holonomy is a closed Wilson loop.) This definition also holds in continuum gauge theory.] When any configuration is gauge transformed by a minimization procedure, such as used here, it is mapped into the Gribov region $\Omega$. In [5] it was shown: When a thin center vortex configuration is gauge transformed into minimal Coulomb gauge it is mapped onto a configuration $U_{0}$ that lies on the boundary $\partial \Omega$ of $\Omega$. Moreover its Faddeev-Popov operator $M\left(U_{0}\right)=-\nabla \cdot \mathcal{D}\left(U_{0}\right)$ has a non-trivial null space that is $\left(N^{2}-1\right)$ dimensional. (This is in addition to the $\left(N^{2}-1\right)$-dimensional trivial null-space consisting of constant eigenvectors. Here and below we do not count trivial eigenvectors that are generators of global gauge transformations.) Likewise, when an abelian configuration is gauge-transformed into the Gribov region, its Faddeev-Popov operator has a non-trivial $R$-dimensional null space, where $R$ is the rank of the group. The reason is that the gauge orbit of a center or abelian configuration is degenerate so the gauge-invariant equation $\mathcal{D}_{i}\left(U_{0}\right) \omega=0$, which holds for $i=1,2,3$ simultaneously, has $N^{2}-1$ or $R$ non-trivial solutions respectively.

\subsection{Tangent plane to Gribov horizon at a regular point}

Let $a_{i}^{b}(x)$ be a set of coordinates of the group element $U_{i}(x)=U\left[a_{i}^{b}(x)\right]$. It is convenient to use coordinates $a_{i}^{b}(x)$ such that the Coulomb gauge condition is linear, $(\nabla \cdot a)^{b}(x)=$ $\sum_{i}\left[a_{i}^{b}(x)-a_{i}^{b}(x-\hat{\imath})\right]=0$, and in terms of which $\Omega_{+}$(see above) is convex. We write $U_{0}+\delta U=U\left(a_{0}+\delta a\right)$. Here $\delta a$ is an arbitrary (transverse) small variation of the coordinates at $a_{0}$. Such a variation is a tangent vector at $a_{0}$, and the space of tangent vectors constitutes the tangent space at $a_{0}$.

Let $U_{0}$ be a configuration in Coulomb gauge, that lies on the boundary $\partial \Omega$ of the Gribov region. By definition, the corresponding Faddeev-Popov operator has a non-trivial null eigenvector

$$
M\left(U_{0}\right) \omega_{0}=0,
$$

all other eigenvalues being non-negative. We are interested in the case of a center configuration in Coulomb gauge where the non-trivial null eigenvalue of $M\left(U_{0}\right)$ is $\left(N^{2}-1\right)$-fold 
degenerate, but for orientation we first consider the case where the non-trivial null eigenvalue is non-degenerate. Let $U_{0}+\delta U$ be a neighboring point that is also on the Gribov horizon, so $M\left(U_{0}+\delta U\right)$ also possesses a null vector

$$
M\left(U_{0}+\delta U\right)\left(\omega_{0}+\delta \omega\right)=0 .
$$

We wish to find the condition on $\delta a$ that holds whenever $U\left(a_{0}+\delta a\right)$ also lies on the Gribov horizon $\partial \Omega$. We have

$$
M\left(U_{0}+\delta U\right)=M\left(U_{0}\right)+\delta M,
$$

where

$$
\delta M=\sum_{x i b} \delta a_{i}^{b}(x) \partial_{x i b} M_{0}
$$

and

$$
\left.\partial_{x i b} M_{0} \equiv \frac{\partial M}{\partial a_{i}^{b}(x)}\right|_{a=a_{0}} .
$$

To first order in small quantities we have

$$
\delta M \omega_{0}+M\left(U_{0}\right) \delta \omega=0 .
$$

We contract this equation with $\omega_{0}$ and obtain

$$
\left(\omega_{0}, \delta M \omega_{0}\right)=0,
$$

or

$$
\sum_{x i b} \delta a_{i}^{b}(x)\left(\omega_{0}, \partial_{x i b} M_{0} \omega_{0}\right)=0 .
$$

Geometrically, this is the statement that $\delta a_{i}^{b}(x)$ is perpendicular to the vector $\left(\omega_{0}, \partial_{x i b} M_{0} \omega_{0}\right)$, so this vector is the normal to $\partial \Omega$ at $U_{0}=U\left(a_{0}\right)$. It defines the a hyperplane that is tangent to the Gribov horizon at $a_{0}$.

\subsection{Center vortices as singularities of the Gribov horizon}

\subsubsection{General idea}

Suppose that $U_{0}=U\left(a_{0}\right)$ is a point on the Gribov horizon and that the null eigenvalue of $M\left(a_{0}\right)$ is $P$-fold degenerate,

$$
M\left(a_{0}\right) \omega_{0}^{(n)}=0 ; \quad n=1, \ldots, P .
$$

As noted above, this happens for every gauge copy in Coulomb gauge of a thin center vortex configuration, where $P=N^{2}-1$. Under the small perturbation $M\left(a_{0}+\delta a\right)=$ $M\left(a_{0}\right)+\delta M$, where $\delta M$ is given above, the $P$-fold degenerate null-space splits into $P$ levels with eigenvalues $\delta \lambda_{n}$, for $n=1, \ldots, P$, that depend linearly, $\delta \lambda_{n}=\sum_{x i a} C_{n, i}^{b}(x) \delta a_{i}^{b}(x)$, on the $\delta a$. 
For a point $a_{0}$ on the boundary $\partial \Omega$ we define the Gribov region of the tangent space at $a_{0}$ to be the set of tangent vectors that point inside $\Omega$. We designate it by $\Omega_{a_{0}}$. More formally, it is the set of tangent vectors $\delta a$ at $a_{0}$ such that $a_{0}+\delta a$ lies in $\Omega$,

$$
\Omega_{a_{0}} \equiv\left\{\delta a: a_{0}+\delta a \in \Omega\right\} .
$$

Recall that by definition the Gribov region $\Omega$ consists of (transverse) configurations $U(a)$ such that all eigenvalues of $M(a)$ are positive. It follows that $\Omega_{a_{0}}$ is the set of $\delta a$ such that all $P$ eigenvalues $\delta \lambda_{n}(\delta a)$ are positive,

$$
\Omega_{a_{0}} \equiv\left\{\delta a: \delta \lambda_{n}(\delta a)>0 \text { for } n=1, \ldots, P\right\} .
$$

This condition is quite restrictive because, for generic $\delta a$, some number $\nu$ of eigenvalues $\delta \lambda_{n}$ are negative while $P-\nu$ are positive, where $\nu=0,1, \ldots, P$. As a result the boundary $\partial \Omega_{a_{0}}$ of the Gribov region at $a_{0}$ is not simply a tangent plane through $a_{0}$, as before. Rather it is a high dimensional wedge-conical vertex whose shape we shall find.

\subsubsection{Degenerate perturbation theory}

The eigenvalues in an infinitesimal neighborhood of a point $U_{0}$ on the Gribov horizon are determined by the eigenvalue equation

$$
\left[M\left(U_{0}\right)+\delta M\right]\left(\sum_{n} c_{n} \omega_{0}^{(n)}+\delta \omega\right)=\delta \lambda\left(\sum_{n} c_{n} \omega_{0}^{(n)}+\delta \omega\right),
$$

or

$$
\delta M \sum_{n} c_{n} \omega_{0}^{(n)}+M\left(U_{0}\right) \delta \omega=\delta \lambda \sum_{n} c_{n} \omega_{0}^{(n)} .
$$

Upon contracting this equation with $\omega_{0}^{(m)}$, we obtain, by $(7.9)$, the $P$-dimensional eigenvalue equation

$$
\sum_{n} \delta a_{m n} c_{n}=\delta \lambda c_{m}
$$

where

$$
\begin{aligned}
\delta a_{m n} & \equiv\left(\omega_{0}^{(m)}, \delta M \omega_{0}^{(n)}\right) \\
& =\sum_{x i b} \delta a_{i}^{b}(x)\left(\omega_{0}^{(m)}, \partial_{x i b} M_{0} \omega_{0}^{(n)}\right) .
\end{aligned}
$$

Abstractly, $\delta a$ is a tangent vector in lattice configuration space at point $a_{0}$ in lattice configuration space; the components of this vector, in a suitable basis, are denoted $\delta a_{i}^{b}(x)$. We may also regard the set of numbers $\delta a_{m n}$ as components of $\delta a$ in some other basis.

The eigenvalue equation has $P$ solutions $\delta \lambda_{n}$. The condition that they all be positive, which determines $\Omega_{a_{0}}$, the Gribov region at $a_{0}$, is the condition that the matrix $\delta a_{m n}$ define a strictly positive form. Such a form is characterized by the Sylvester criterion that $\operatorname{det} \delta a_{m n}$ be positive, together with the determinant of all principle minors (diagonal square submatrices) of this matrix. The boundary of this region is determined by the condition that one eigenvalue vanish, which happens when the determinant vanishes,

$$
\operatorname{det} \delta a_{m n}=0 .
$$




\subsubsection{Two-fold degeneracy}

We first analyze 2-fold degeneracy. In this case positivity of the two principle minors and of the determinant reads

$$
\delta a_{11}>0, \quad \delta a_{22}>0, \quad \delta a_{11} \delta a_{22}-\delta a_{12}^{2}>0 .
$$

In terms of $\delta a_{+} \equiv \frac{1}{2}\left(\delta a_{11}+\delta a_{22}\right)$ and $\delta a_{-} \equiv \frac{1}{2}\left(\delta a_{11}-\delta a_{22}\right)$, the last condition reads

$$
\delta a_{+}^{2}-\delta a_{-}^{2}-\delta a_{12}^{2}>0
$$

The three inequalities define the interior of the "future" cone in the 3 -variables $\delta a_{+}, \delta a_{-}$ and $\delta a_{12}$, with vertex at the origin. Thus the boundary of the Gribov region at $a_{0}$ is a cone in these 3 variables. Taking account of the remaining components of $\delta a$, the conical singularity can be viewed as a kind of wedge in higher dimensions.

\subsubsection{Three-fold degeneracy}

For $\mathrm{SU}(2)$ gauge theory the thin-vortex configurations are 3-fold degenerate points on the Gribov horizon. In this case the positivity of the principle minors and the determinants reads

$$
\begin{gathered}
\delta a_{11}>0, \quad \delta a_{22}>0, \quad \delta a_{33}>0 \\
\delta a_{11} \delta a_{22}-\delta a_{12}^{2}>0 \\
\delta a_{11} \delta a_{33}-\delta a_{13}^{2}>0 \\
\delta a_{22} \delta a_{33}-\delta a_{23}^{2}>0 \\
\operatorname{det} \delta a_{m n}>0 .
\end{gathered}
$$

These 7 inequalities characterize the Gribov region $\Omega_{a_{0}}$ of the tangent space at a point $a_{0}$ on the Gribov horizon that is 3 -fold degenerate.

From our discussion of 2-fold degeneracy we know that the positivity of each of the 2 by 2 determinants and of its diagonal elements defines a "future" cone. We call these future cones $F_{12}, F_{13}$ and $F_{23}$ respectively. For example $F_{12}$ is defined by $\delta a_{11}>0$, and $\delta a_{22}>0$, and

$$
\left[\frac{1}{2}\left(\delta a_{11}+\delta a_{22}\right)\right]^{2}-\left[\frac{1}{2}\left(\delta a_{11}-\delta a_{22}\right)\right]^{2}-\delta a_{12}^{2}>0,
$$

etc. Each is a cone of opening half-angle $\pi / 4$, and we have shown that $\Omega_{a_{0}}$ is contained in the intersection of the 3 future cones,

$$
\Omega_{a_{0}} \subset F_{12} \cap F_{13} \cap F_{23} .
$$

This condition and the 3 by 3 determinantal inequality $\operatorname{det} \delta a_{m n}>0$ characterize the Gribov region $\Omega_{a_{0}}$ in the tangent space of a point $U_{0}=U\left(a_{0}\right)$ that is 3 -fold degenerate. 


\subsubsection{Over-all picture of the Gribov horizon and its center-vortex singularities}

We have seen that $\Omega_{+}$is convex and that thin center vortex configurations are wedgeconical singularities on the boundary of $\partial \Omega$. Those that are on $\partial \Omega_{+}$are extremal elements, like the tips on a very high-dimensional pineapple. Indeed, each center configuration is an isolated point. If one moves a small distance from a center configuration it is no longer a center configuration. Its gauge transform $U\left(a_{0}\right)$ in Coulomb gauge is likewise an isolated point on the Gribov horizon. Thus the wedge in the boundary $\partial \Omega$ at $a_{0}$ that we have just described occurs at an isolated point where the Gribov horizon may be said to have a "pinch".

In $\mathrm{SU}(2)$ gauge theory there are $2^{d V}$ center configurations (where $d$ is the number of dimensions of space, and $V$ is the volume of the lattice) because there are $d V$ links in the lattice and there are 2 center elements in $\mathrm{SU}(2)$. These are related by $2^{V}$ gauge transformations, so there are $2^{(d-1) V}$ center orbits. The absolute minimum of each of these orbits lies on the common boundary of the fundamental modular region $\partial \Lambda$ and the Gribov region $\partial \Omega$. So there are at least $2^{(d-1) V}$ tips on the above-mentioned pineapple, a truly enormous number. Moreover for each such orbit there are many Gribov copies, all lying on $\partial \Omega$ (spin glass problem). These are all singular points of the Gribov horizon of the type described. For $\mathrm{SU}(2)$ there may not be any other singular points on $\partial \Omega$. It is possible that the thin center vortex configurations provide a rather fine triangulation of $\partial \Omega$.

Note: The gauge transform in Coulomb gauge of an abelian configuration also lies on the Gribov horizon. However the $\mathrm{SU}(2)$ group is of rank 1, so, for $\mathrm{SU}(2)$ gauge theory, such a configuration is invariant under a one-parameter group of transformations only. As a result, the corresponding null space $M\left(U_{0}\right) \omega_{0}=0$ is only one-dimensional, and the present considerations do not indicate that these are singularities of $\partial \Omega$.

\section{Coulomb gauge as an attractive fixed point of the interpolating gauge}

The data reported above have been obtained by numerically gauge fixing to the minimal Coulomb gauge using a well-defined numerical minimization procedure on a lattice of finite volume. However not every lattice gauge corresponds to a well-defined continuum gauge, so one may well ask whether our results have a continuum analog, especially since in continuum theory the Coulomb gauge is a singular gauge. Indeed, in Coulomb gauge, some propagators of the form $1 / \mathbf{k}^{2}$ appear instead of $1 /\left(k_{0}^{2}+\mathbf{k}^{2}\right)$, which leads to unrenormalizable

divergences $\int d k_{0}$ in some closed loops. This has been treated by using the action in phase space (first order formalism), which allows a systematic cancellation of these divergences [18], or by using an interpolating gauge [19] with gauge condition

$$
a \partial_{0} A_{0}+\nabla \cdot \mathbf{A}=0
$$

This gauge interpolates between the Landau gauge $a=1$ and the Coulomb gauge, $a=0$, and may also be achieved by a numerical minimization procedure. For $a>0$ it is a regular continuum gauge, and the gauge parameter $a$ provides a regularization of the divergences of the Coulomb gauge, which is obtained in the limit $a \rightarrow 0$ of the interpolating gauge. 
However as a possible obstacle to this regularization, it must be noted that the quantities that appear in the interpolating gauge condition (8.1) are unrenormalized quantities, and the unrenormalized gauge parameter depends on the ultraviolet cut-off $\Lambda$,

$$
a=a\left(\Lambda / \Lambda_{\mathrm{QCD}}\right) .
$$

For the Coulomb-gauge limit of the interpolating gauge to be a smooth limit it is necessary that the gauge parameter $a\left(\Lambda / \Lambda_{\mathrm{QCD}}\right)$ flows toward (or at least not away from) the Coulombgauge value $a=0$, as the ultraviolet cut-off is removed, $\Lambda \rightarrow \infty$. In Appendix $\mathbb{Q}$ its dependence on $\Lambda$ is calculated in the neighborhood of $a=0$ to one-loop order in the perturbative renormalization group, with the result in pure $\mathrm{SU}(N)$ gauge theory,

$$
a\left(\Lambda / \Lambda_{\mathrm{QCD}}\right)=\frac{\text { const }}{\left[\ln \left(\Lambda / \Lambda_{\mathrm{QCD}}\right)\right]^{4 / 11}},
$$

This gives

$$
\lim _{\Lambda \rightarrow \infty} a\left(\Lambda / \Lambda_{\mathrm{QCD}}\right)=0,
$$

and we conclude that in some neighborhood of $a=0$, the Coulomb gauge value $a=0$ is an attractive fixed point of the renormalization-group flow. This removes the possible obstacle to regularization of the Coulomb gauge by the interpolating gauge.

[Similarly the renormalized gauge parameter $a^{R}=a^{R}\left(\mu / \Lambda_{\mathrm{QCD}}\right)$, depends on the renormalization mass $\mu$. As the renormalization mass $\mu$ gets large, which is the appropriate choice for calculations at high momentum, one has at one-loop order

$$
a^{R}\left(\mu / \Lambda_{\mathrm{QCD}}\right)=\frac{\text { const }}{\left[\ln \left(\mu / \Lambda_{\mathrm{QCD}}\right)\right]^{4 / 11}} .
$$

So again, the Coulomb-gauge value $a^{R}=0$ is an attractive fixed point of the renormalizationgroup flow.]

\section{Conclusions}

We have found that the low-lying eigenvalues of the Faddeev-Popov operator, in thermalized lattices, tend towards zero as the lattice volume increases. This means that in the infinite volume limit, thermalized configurations lie on the Gribov horizon. That fact alone would not allow us to make any strong conclusions about the energy of unscreened color charge. However, the data also indicate that the density $\rho(\lambda)$ of F-P eigenvalues goes as a small power of $\lambda$, at infinite volume, as $\lambda \rightarrow 0$. Together with the behavior of $F(\lambda)$ at $\lambda \rightarrow 0$, we conclude that the energy of an unscreened color charge is infrared divergent, and that this divergence can be attributed to the near-zero modes of the Faddeev-Popov operator.

This evidence clearly supports the Gribov horizon scenario advocated by Gribov and Zwanziger in ref. [2]. This scenario was invented to account for confinement, and the reader may be surprised to find a linearly rising color-Coulomb potential in the deconfined phase. However this is nicely explained by the horizon scenario in Coulomb gauge, as 
we now explain. In Coulomb gauge the gauge fixing is done independently on each 3dimensional time slice, and may be done on a single time slice. According to the horizon scenario, on each time slice, 3-dimensional configurations $A_{i}(\mathbf{x})$ are favored that lie near the horizon of a 3-dimensional gauge theory, and this enhances the instantaneous colorCoulomb potential. This is true for every temperature $T$, including in the deconfined phase, because temperature determines the extent of the lattice in the fourth dimension. Thus, the horizon scenario provides a framework in which confinement may be understood, but it is not detailed enough to tell us under what conditions the infinite color-Coulomb potential may be screened to give a finite self-energy, as measured by the Polyakov loop.

By factoring thermalized lattices into vortex-only and vortex-removed components, we have also been able to show that the constant density of low-lying F-P eigenvalues can be entirely attributed to the vortex component. We find that the eigenvalue density of the vortex component is qualitatively similar to that of the full configuration. The eigenvalue density of the vortex-removed component, on the other hand, is drastically different from that of the full configuration. This density can be interpreted as simply a small perturbation of the zero-field (or zero-th order) result, and it is identical in form to the (non-confining) eigenvalue density of lattice configurations in the Higgs phase of a gauge-Higgs theory.

These findings establish a firm connection between the center vortex and the Gribov horizon confinement scenarios. According to the center vortex doctrine, fluctuations in the vortex linking number are responsible for the area law falloff of Wilson loops. It now appears that vortex configurations are also responsible for the enhanced density of near-zero F-P eigenvalues, which is essential to the Gribov horizon picture. This result is consistent with previous results in ref. [20], where it was found that vortex removal also removes the Coulomb string tension of the color Coulomb potential. It is also consistent with recent investigations of Gattnar, Langfeld, and Reinhardt [21] in Landau gauge.

The F-P eigenvalue spectrum at high temperatures, with and without vortices, turns out quite similar to the corresponding results at low-temperature. This similarity was to be expected, since the F-P operator depends only on spacelike links at a fixed time, and even at high $T$ these form a confining three-dimensional ensemble for spacelike Wilson loops. The Gribov scenario must therefore be relevant to physics in the deconfined phase; cf. ref. 22] for a recent application.

We also report a result which supports the consistency of Coulomb gauge itself in the continuum limit. Coulomb gauge is very singular in continuum perturbation theory, and one method of making it better defined is to view Coulomb gauge as a non-singular limit of the more general gauge condition $a \partial_{0} A_{0}+\nabla \cdot \mathbf{A}=0$. The success of this approach depends on whether the Coulomb gauge limit, $a=0$, is an attractive ultraviolet fixed point of the renormalization group flow. Here we have shown that this requirement is satisfied.

Finally, we have uncovered an intriguing geometrical property of thin vortices in lattice configuration space. In ref. [5] it was shown that thin vortices (gauge equivalent to center configurations) lie on the Gribov horizon. The Gribov horizon is a convex manifold, and we have shown here that thin vortices are conical singularities on that manifold. Percolating thick vortices appear to be ubiquitous in thermalized lattice configurations at or near the Gribov horizon; it is conceivable that the special geometrical status of thin vortices is in 
some way related to the ubiquity of their finite-thickness cousins.

\section{Acknowledgments}

J.G. thanks Poul Henrik Damgaard for helpful discussions on the scaling of matrix model eigenvalue distributions.

Our research is supported in part by the U.S. Department of Energy under Grant No. DE-FG03-92ER40711 (J.G.), the Slovak Science and Technology Assistance Agency, Grant No. APVT-51-005704 (Š.O.), and the National Science Foundation, Grant No. PHY-0099393 (D.Z.).

\section{A. Finite-volume scaling of low-lying F-P eigenvalues}

In $N \times N$ random matrix models, the tail of the eigenvalue distribution often displays a universal scaling behavior with $N$. This fact has found important applications in the study of chiral symmetry breaking, where this sort of universal scaling is found in the density of near-zero eigenvalues of the Euclidean Dirac operator as a function of lattice volume (which is proportional to the number of eigenvalues) [23]. In our case, we are also interested in the density of near-zero eigenvalues in the infinite 3 -volume limit. The statement in this case is as follows (cf., e.g., ref. [24]): Suppose the normalized density of low-lying eigenvalues, at very large volumes, goes as

$$
\rho(\lambda)=\kappa \lambda^{\alpha}
$$

where $\kappa$ is some constant. Then the density of eigenvalues, the average spacing between the low-lying eigenvalues, and the probability density $P\left(\lambda_{n}\right)$ of the $n$-th low-lying eigenvalue, agree for every lattice 3 -volume $V_{3}$, if the eigenvalues themselves are rescaled according to

$$
z=\lambda V_{3}^{\frac{1}{1+\alpha}}
$$

The argument goes as follows: The number of eigenvalues $N[\lambda, \Delta \lambda]$ in the interval $[\lambda-$ $\left.\frac{1}{2} \Delta \lambda, \lambda+\frac{1}{2} \Delta \lambda\right]$ is

$$
N[\lambda, \Delta \lambda]=3 V_{3} \rho(\lambda) \Delta \lambda .
$$

Then in terms of rescaled eigenvalues $z=\lambda V_{3}^{p}$, we have

$$
N[\lambda, \Delta \lambda]=3 \kappa V_{3}^{1-p(1+\alpha)} z^{\alpha} \Delta z .
$$

If we require that this number of eigenvalues depends only on the rescaled variables $z, \Delta z$, then it is necessary that $p=1 /(1+\alpha)$, and eq. (A.2) follows.

Our strategy is to plot the probability density $P\left(\lambda_{\text {min }}\right)$, rescaled by a factor $V_{3}^{-1 /(1+\alpha)}$, as a function of the variable $z_{\min }=\lambda_{\min } V_{3}^{1 /(1+\alpha)}$, where $\lambda_{\min }$ is the lowest non-zero eigenvalue. The rescaling of the probability density ensures that its integral over $z_{\text {min }}$ is unity. $P\left(\lambda_{\min }\right)$ is computed on $8^{4}$ to $20^{4}$ lattice volumes, at various values of $\alpha$. If we can find a value of $\alpha$ for which the (rescaled) probability densities $P\left(\lambda_{\min }\right)$ coincide at all lattice sizes, then this determines $\alpha$, and in turn the behavior of the eigenvalue density near $\lambda=0$. 
The results for rescaled $P\left(\lambda_{\min }\right)$, for both the full configurations $\left(\lambda_{\min }=\lambda_{4}\right)$, and the vortex-only configurations $\left(\lambda_{\min }=\lambda_{7}\right)$, are displayed in Fig. 17. We show only three values of $\alpha$ in each case, which include our best estimate for the scaling $\alpha$. We find that $\alpha=0.25 \pm 0.05$ for the full configurations, and $\alpha=0.0 \pm 0.05$ for the vortex-only configurations seem to give the best scaling results; the error estimate is subjective.

The lattice data for $L=20$ in the vortex-only configurations calls for some comment. This distribution does not match the distributions obtained at smaller $L$ at any $\alpha$. This could mean that the scaling hypothesis for low-lying eigenvalues is invalid, but in our opinion the mismatch at $L=20$ has a different explanation: We believe the problem is connected to difficulties we have encountered with gauge fixing on these large lattices. As lattice size is increased, typical values of $\lambda_{\min }$ become smaller, and the number of overrelaxation steps required to satisfy our Coulomb gauge convergence criterion increases. In general, on a given lattice size, the number of over-relaxation steps required to gauge fix is inversely correlated with the size of $\lambda_{\min }$ in the gauge-fixed configuration.

It happens occasionally that even after 10,000 gauge-fixing steps on a given time-slice, the spatial lattice configuration has not converged to Coulomb gauge. When this happens, we perform a random gauge transformation at that time slice and gauge fix a second time, to a different Gribov copy (note that in Coulomb gauge, time-slices can be, and were, gaugefixed independently). This procedure most likely biases the result towards higher average values of $\lambda_{\min }$. In doing things this way, we are almost certainly modifying the probability distribution in Gribov region, giving lower measure to Gribov copies which are closer to the horizon. On the other hand, simply excluding these hard-to-gauge-fix lattices would probably introduce an even worse bias. On smaller $(L<20)$ vortex-only lattices, and on the full, unmodified lattices, problems with convergence to Coulomb gauge are uncommon. However, on the $L=20$ vortex-only lattice, there is a convergence failure on almost $38 \%$ of all time slices; on these slices we have performed a random gauge transformation to move to a different Gribov copy. The rate of convergence failure on vortex-only $16^{4}$ lattices is five times lower, and the rate of failure on unmodified $20^{4}$ lattices is eight times lower, than on the vortex-only $20^{4}$ lattice. For this reason, we believe that the bias towards larger eigenvalues is by far the worst on the vortex-only $20^{4}$ lattices, and this is in fact where we see the mismatch, in $P\left(\lambda_{\min }\right)$ vs. $z_{\min }$ at $\alpha \approx 0$, with the other vortex-only lattice volumes.

Note that for the vortex-only configurations, $\alpha=0$ is consistent with a finite, non-zero value for $\rho(0)$, as shown in eq. (2.25). On the other hand, it is not excluded that $\alpha$ could in fact be slightly negative, in which case $\rho(\lambda)$ actually diverges at $\lambda=0$. Since the eigenvalue density in Fig. $⿴$ does appear to actually rise as $\lambda \rightarrow 0$, before suddenly falling, a divergent behavior at $\lambda=0$ in the infinite volume limit is not at all excluded.

Next we consider $F(\lambda)$ as $\lambda \rightarrow 0$. We have fit the average value of $F$ at the lowest non-zero eigenvalue, $\left\langle F\left(\lambda_{\min }\right)\right\rangle$, to the form

$$
\left\langle F\left(\lambda_{\min }\right)\right\rangle=\frac{a}{L^{p}}+b .
$$

Our result, within errorbars, is consistent with

$$
\left\langle F\left(\lambda_{4}\right)\right\rangle=\frac{10}{L} \quad \text { full configurations, }
$$




\section{Rescaled $\mathrm{P}\left(\lambda_{\min }\right)$}

Full configurations
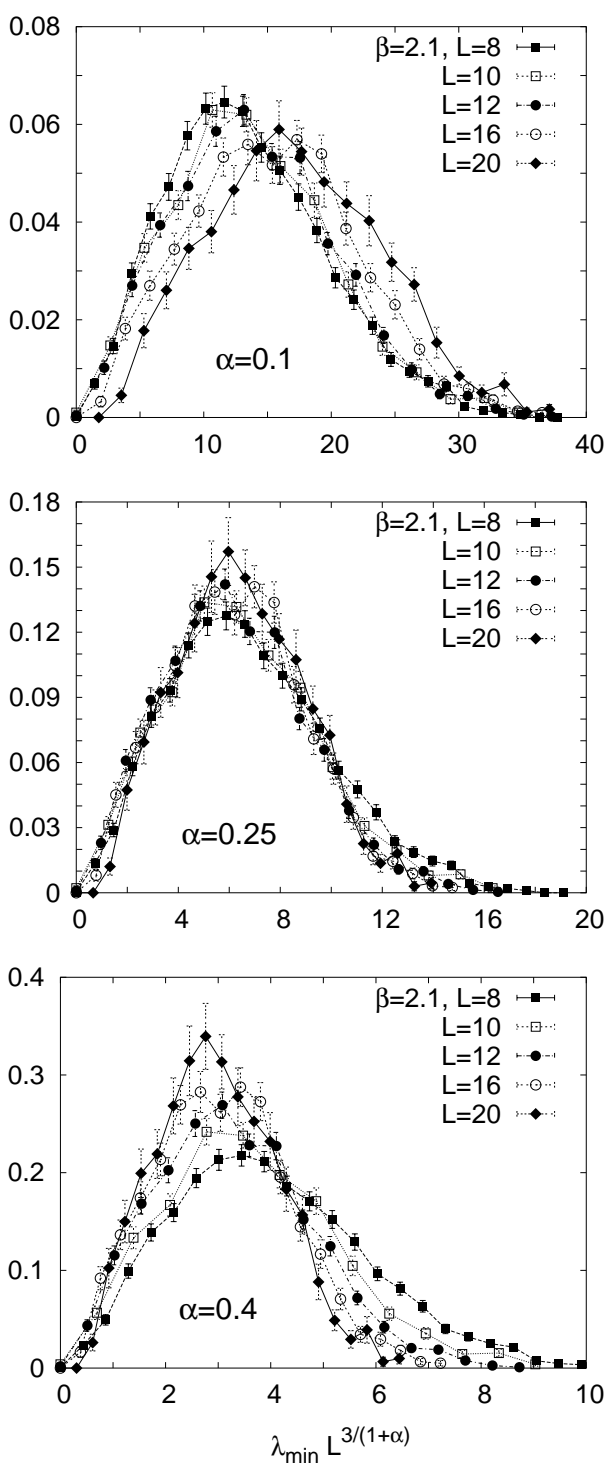

Vortex-only configurations
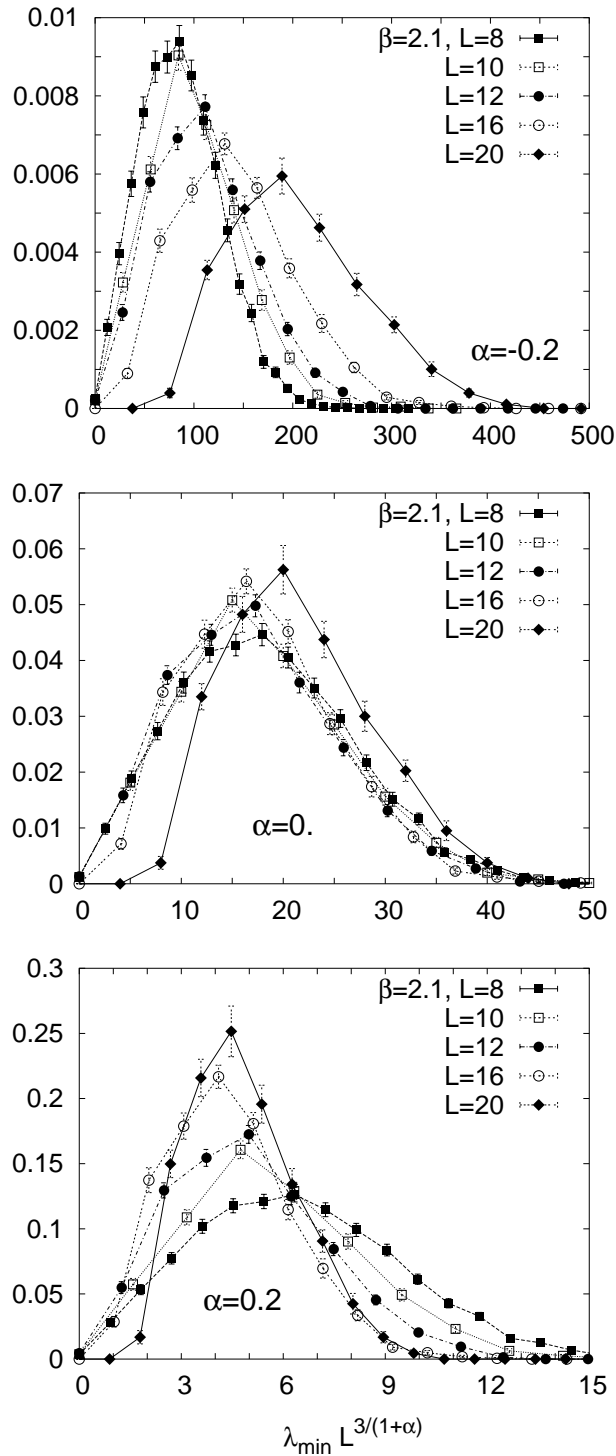

Figure 17: Probability distribution of the values of the lowest non-trivial FP eigenvalue $\lambda_{\min }$ on a variety of lattice sizes $8^{4}-20^{4}$, as a function of the rescaled eigenvalue $z_{\min }=$ $\lambda_{\min } L^{3 / 1+\alpha}$. Results for the full configurations are in the left-hand column of figures, with the vortex-only results in the right-hand column. Each figure corresponds to a different choice of $\alpha ; \alpha=0.1,0.25 .0 .4$ for the full configurations, $\alpha=-0.2,0.0,0.2$ for the vortexonly configurations. The closest match of rescaled $P\left(\lambda_{\min }\right)$ at different volumes comes at $\alpha \approx 0.25$ for the full configurations, and $\alpha \approx 0$ for vortex only configurations (with the exception of $L=20$, see text). 


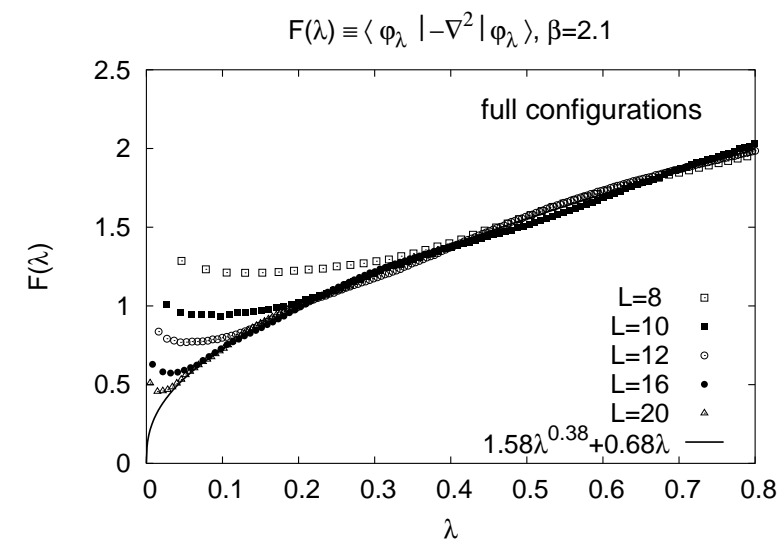

Figure 18: $F(\lambda)$ as in Fig. 2, together with a best fit to eq. (A.8).

$$
\left\langle F\left(\lambda_{7}\right)\right\rangle=1+\frac{20}{L^{1.4}} \quad \begin{gathered}
\text { vortex-only } \\
\text { configurations. }
\end{gathered}
$$

Since $\lambda_{\min } \rightarrow 0$ at infinite volume, then $F(0)=1$ for vortex-only configurations, as stated in eq. (2.25). For the full configurations, since we estimate $\alpha \approx 0.25$, with the consequence that $\left\langle\lambda_{\min }\right\rangle \sim 1 / L^{2.4}$, it is reasonable to guess that for the full configurations near $\lambda=0$,

$$
F(\lambda) \sim \lambda^{0.42}
$$

We have therefore tried the following fit

$$
F(\lambda)=a \lambda^{p}+b \lambda
$$

to the $L=20$ data, where the linear term is motivated from the fact that perturbative behavior (linear dependence on $\lambda$ ) is expected at large $\lambda$. The fit gives the result shown in Fig. 18, with an exponent $p=0.38$ which is not far off our guess of $p=0.42$.

\section{B. Proof of convexity of FMR in SU(2) lattice gauge theory}

The defining property (6.2) of the fundamental modular region may be written

$$
\sum_{x i} \operatorname{Re} \operatorname{Tr}\left[U_{i}(x) g_{i}(x)\right] \leq \sum_{x i} \operatorname{Re} \operatorname{Tr} U_{i}(x),
$$

where $g_{i}(x) \equiv g(x+\hat{\imath}) g(x)^{-1}$. We write $g_{i}(x)=c_{i}(x)+i \vec{\sigma} \cdot \vec{g}_{i}(x)$, where $c_{i}(x)= \pm[1-$ $\left.\vec{g}_{i}(x)^{2}\right]^{1 / 2}$, and the defining property reads,

$$
-\sum_{x i} \vec{a}_{i}(x) \cdot \vec{g}_{i}(x) \leq \sum_{i x} b_{i}(x)\left[1-c_{i}(x)\right],
$$

where the last factor is positive, $1-c_{i}(x) \geq 0$. Given that this property holds for configurations $a_{1}$ and $a_{2}$,

$$
-\sum_{x i} \vec{a}_{1, i}(x) \cdot \vec{g}_{i}(x) \leq \sum_{x i} b_{1, i}(x)\left[1-c_{i}(x)\right]
$$




$$
-\sum_{x i} \vec{a}_{2, i}(x) \cdot \vec{g}_{i}(x) \leq \sum_{x i} b_{2, i}(x)\left[1-c_{i}(x)\right]
$$

then we will show that it holds for $a \equiv \alpha a_{1}+\beta a_{2}$, as stated in section V. Upon multiplying the first equation by $\alpha$ and the second by $\beta$ and adding we obtain,

$$
-\sum_{x i} \vec{a}_{i}(x) \cdot \vec{g}_{i}(x) \leq \sum_{x i}\left[\alpha b_{1, i}(x)+\beta b_{2, i}(x)\right]\left[1-c_{i}(x)\right]
$$

where $\vec{a}_{i}(x) \equiv \alpha \vec{a}_{1, i}(x)+\beta \vec{a}_{2, i}(x)$. It follows from this that the defining property (B.2) that we wish to establish will be proven if we can show that on each link $(x i)$ the inequality

$$
\alpha b_{1, i}(x)+\beta b_{2, i}(x) \leq b_{i}(x)
$$

holds, where $b_{i}(x)=\left[1-\vec{a}_{i}(x)^{2}\right]^{1 / 2}$.

We introduce trigonometric functions

$$
\begin{aligned}
b_{1, i}(x)=\cos \theta_{1, i}(x) ; & \vec{a}_{1, i}(x)=\sin \theta_{1, i}(x) \hat{n}_{1, i}(x), \\
b_{2, i}(x)=\cos \theta_{2, i}(x) ; & \vec{a}_{2, i}(x)=\sin \theta_{2, i}(x) \hat{n}_{2, i}(x), \\
b_{i}(x)=\cos \theta_{i}(x) ; & \vec{a}_{i}(x)=\sin \theta_{i}(x) \hat{n}_{i}(x),
\end{aligned}
$$

where $0 \leq \theta_{1, i}(x) \leq \pi / 2$, and $0 \leq \theta_{2, i}(x) \leq \pi / 2$, and $0 \leq \theta_{i}(x) \leq \pi / 2$. They are related by

$$
\alpha \sin \theta_{i}(x) \hat{n}_{1, i}(x)+\beta \sin \theta_{2, i}(x) \hat{n}_{2, i}(x)=\sin \theta_{i}(x) \hat{n}_{i}(x),
$$

and the property to be proved reads

$$
\alpha \cos \theta_{1, i}(x)+\beta \cos \theta_{2, i}(x) \leq \cos \theta_{i}(x) .
$$

We have

$$
\alpha \cos \theta_{1, i}(x)+\beta \cos \theta_{2, i}(x) \leq \cos \left[\alpha \theta_{1, i}(x)+\beta \theta_{2, i}(x)\right]
$$

by the convexity of the cosine function, so it is sufficient to prove

$$
\cos \left[\alpha \theta_{1, i}(x)+\beta \theta_{2, i}(x)\right] \leq \cos \theta_{i}(x) .
$$

The triangle inequality applied to (B.10) reads

$$
\begin{aligned}
\sin \theta_{i}(x) & \leq \alpha \sin \theta_{1, i}(x)+\beta \sin \theta_{2, i}(x) \\
& \leq \sin \left[\alpha \theta_{1, i}(x)+\beta \theta_{2, i}(x)\right]
\end{aligned}
$$

where we have also used the convexity of the sine function. This yields the inequality $\theta_{i}(x) \leq \alpha \theta_{1, i}(x)+\beta \theta_{2, i}(x)$, from which (B.11) follows. This proves the convexity of $\Lambda_{+}$. 


\section{Renormalization-group flows toward Coulomb gauge}

We compute the flow $^{5}$ of the gauge parameter $a$ that appears in the interpolating gauge condition (8.1) in the neighborhood of $a=0$.

Lorentz-invariance is not manifest in the interpolating gauge, and $A_{0}$ and $\mathbf{A}$ renormalize independently,

$$
A_{0}=Z_{A_{0}} A_{0}^{R} ; \quad \mathbf{A}=Z_{\mathbf{A}} \mathbf{A}^{R}
$$

where superscript $R$ designates renormalized quantities. The renormalized gauge parameter $a^{R}$ is defined by

$$
a=Z_{a} a^{R}
$$

where the renormalization constant $Z_{a}$ is determined by the renormalized gauge condition,

$$
a^{R} \partial_{0} A_{0}^{R}+\nabla \cdot \mathbf{A}^{R}=0 .
$$

This gives

$$
Z_{a}=Z_{A_{0}}^{-1} Z_{\mathbf{A}}
$$

The $\gamma$-functions are defined by

$$
\begin{aligned}
\partial_{t} \ln Z_{a} & =\gamma_{a}(g), \\
\partial_{t} \ln Z_{A_{0}} & =\gamma_{A_{0}}(g), \\
\partial_{t} \ln Z_{\mathbf{A}} & =\gamma_{\mathbf{A}}(g),
\end{aligned}
$$

where $t \equiv \ln \Lambda$, and $\Lambda$ is the ultraviolet cut-off. The derivative is taken at fixed renormalized coupling constant $g^{R}$. We have

$$
\partial_{t} \ln Z_{a}=-\partial_{t} \ln Z_{A_{0}}+\partial_{t} \ln Z_{\mathbf{A}}
$$

which gives

$$
\gamma_{a}(g)=-\gamma_{A_{0}}(g)+\gamma_{\mathbf{A}}(g) .
$$

From the perturbative expansions

$$
\begin{aligned}
Z_{a} & =1+c_{a} \ln \Lambda\left(g^{R}\right)^{2}+\ldots \\
Z_{A_{0}} & =1+c_{A_{0}} \ln \Lambda\left(g^{R}\right)^{2}+\ldots \\
Z_{\mathbf{A}} & =1+c_{\mathbf{A}} \ln \Lambda\left(g^{R}\right)^{2}+\ldots
\end{aligned}
$$

we obtain

$$
\begin{aligned}
\gamma_{a} & =c_{a} g^{2}+\ldots, \\
\gamma_{A_{0}} & =c_{A_{0}} g^{2}+\ldots, \\
\gamma_{\mathbf{A}} & =c_{\mathbf{A}} g^{2}+\ldots,
\end{aligned}
$$

\footnotetext{
${ }^{5}$ The calculation reported here was motivated by correspondence with Kurt Langfeld and Laurent Moyaerts.
} 
and

$$
c_{a}=-c_{A_{0}}+c_{\mathbf{A}} .
$$

To one-loop order the renormalization constant satisfies

$$
\partial_{t} \ln Z_{a}=c_{a} g^{2}+\ldots=\frac{c_{a}}{2 b_{0} t}+\ldots,
$$

where $b_{0}$ is the first coefficient of the $\beta$-function. In pure $\mathrm{SU}(N)$ gauge theory it has the value

$$
b_{0}=\frac{11}{3} \frac{N}{16 \pi^{2}} \text {. }
$$

We obtain, to one-loop order,

$$
Z_{a}=\text { const } t^{c_{a} / 2 b_{0}},
$$

which, with $t=\ln \Lambda$, gives the leading dependence of the bare gauge parameter on the cut-off $\Lambda$,

$$
a\left(\Lambda / \Lambda_{\mathrm{QCD}}\right)=\text { const }\left(\Lambda / \Lambda_{\mathrm{QCD}}\right)^{c_{a} / 2 b_{0}} .
$$

Clearly $a=0$ is a fixed point of the renormalization group. To see if it is also a stable fixed point, we may evaluate the coefficient $c_{a}$ at $a=0$, namely in Coulomb gauge, assuming that $c_{a}$ is smooth in the neighborhood of $a=0$. The renormalization constants $Z_{A_{0}}$ and $Z_{\mathbf{A}}$ in Coulomb gauge, are given to one-loop order in eq. (B.37) of [18]. The coefficients have the values

$$
c_{A_{0}}=\frac{11}{6} \frac{N}{8 \pi^{2}} \quad c_{\mathbf{A}}=\frac{1}{2} \frac{N}{8 \pi^{2}},
$$

which gives

$$
c_{a}=-\frac{4}{3} \frac{N}{8 \pi^{2}}
$$

and

$$
a\left(\Lambda / \Lambda_{\mathrm{QCD}}\right)=\frac{\text { const }}{\left[\ln \left(\Lambda / \Lambda_{\mathrm{QCD}}\right)\right]^{4 / 11}} .
$$

\section{References}

[1] J. Greensite, The confinement problem in lattice gauge theory, Prog. Part. Nucl. Phys. 51 (2003) 1 hep-lat/0301023.

[2] V. Gribov, Quantization of non-Abelian gauge theories, Nucl. Phys. B 139 (1978) 1;

D. Zwanziger, Renormalization in the Coulomb gauge and order parameter for confinement in QCD, Nucl. Phys. B 518 (1998) 237.

[3] D. Zwanziger, No confinement without Coulomb confinement, Phys. Rev. Lett. 90 (2003) 102001 hep-lat/0209105.

[4] Ph. de Forcrand and M. D'Elia, Relevance of center vortices to QCD, Phys. Rev. Lett. 82 (1999) 4582 hep-lat/9901020.

[5] J. Greensite, Š. Olejník, and D. Zwanziger, Coulomb energy, remnant symmetry, and the phases of non-Abelian gauge theories, Phys. Rev. D 69 (2004) 074506 hep-lat/0401003. 
[6] M. Semenov-Tyan-Shanskii and V. Franke, A variational principle for the Lorentz condition and restriction of the domain of path integration in non-Abelian gauge theory, Zap. Nauch. Sem. Leningrad. Otdeleniya Matematicheskogo Instituta im. V. A. Steklova, AN SSSR, vol. 120, p. 159, 1982 (English translation: New York, Plenum Press 1986).

[7] M. Lavelle and D. McMullan, Hadrons without strings, Phys. Lett. B 471 (1999) 65 hep-ph/9910398.

[8] http://www.caam.rice.edu/software/ARPACK.

[9] C. Lang, C. Rebbi, and M. Virasoro, The phase structure of a non-abelian gauge Higgs field system, Phys. Lett. B 104 (1981) 294.

[10] K. Osterwalder and E. Seiler, Gauge field theories on a lattice, Ann. Phys. (NY) 110 (1978) 440 .

[11] E. Fradkin and S. Shenker, Phase diagrams of lattice gauge theories with Higgs fields, Phys. Rev. D 19 (1979) 3682.

[12] J. Kertész, Existence of weak singularities when going around the liquid-gas critical point, Physica A 161 (1989) 58.

[13] R. Bertle, M. Faber, J. Greensite, and Š. Olejník, Center dominance in SU(2) gauge-Higgs theory, Phys. Rev. D 69 (2004) 014007 hep-lat/0310057.

[14] K. Langfeld, Confinement versus color superconductivity: A lattice investigation, hep-lat/0109033.

[15] K. Langfeld, Lattice effective theory and the phase transition at finite densities, hep-lat/0212032.

[16] C. Borgs, Area law for spatial Wilson loops in high-temperature lattice gauge theories, Nucl. Phys. B 261 (1985) 455; E. Manousakis and J. Polonyi, Nonperturbative length scale in high-temperature QCD, Phys. Rev. Lett. 58 (1987) 847; G. Bali, J. Fingberg, U. Heller, F. Karsch, and K. Schilling, Spatial string tension in the deconfined phase of $(3+1)$-dimensional SU(2) gauge theory, Phys. Rev. Lett. 71 (1993) 3059 hep-lat/9306024; L. Kärkkäinen, P. Lacock, D. Miller, B. Petersson and T. Reisz, Spacelike Wilson loops at finite temperature, Phys. Lett. B 312 (1993) 173 hep-lat/9306015.

[17] M. Engelhardt, K. Langfeld, H. Reinhardt, and O. Tennert, Deconfinement in SU(2) Yang-Mills theory as a center vortex percolation transition, Phys. Rev. D 61 (2000) 054504 hep-lat/9904004; R. Bertle, M. Faber, J. Greensite, and S. Olejník, The structure of projected center vortices in lattice gauge theory, J. High Energy Phys. 03 (1999) 019. hep-lat/9903023; Ph. de Forcrand and L. von Smekal, 't Hooft loops and consistent order parameters for confinement, Nucl. Phys. 106 (Proc. Suppl.) (2002) 619 hep-lat/0110135.

[18] D. Zwanziger, Renormalization in the Coulomb gauge and order parameter for confinement in QCD, Nucl. Phys. B 518 (1998) 237.

[19] L. Baulieu and D. Zwanziger, Renormalizable non-covariant gauges and Coulomb gauge limit, Nucl. Phys. B 548 (1999) 527 hep-th/9807024.

[20] J. Greensite and Š. Olejník, Coulomb energy, vortices, and confinement, Phys. Rev. D 67 (2003) 094503 hep-lat/0302018.

[21] J. Gattnar, K. Langfeld, and H. Reinhardt, Signals of confinement in Green functions of SU(2) Yang-Mills theory, Phys. Rev. Lett. 93 (2004) 061601 hep-lat/0403011. 
[22] D. Zwanziger, Equation of state of gluon plasma from fundamental modular region, hep-ph/0407103.

[23] M. Berbenni-Bitsch, S. Meyer, A. Schäfer, J. Verbaarschot, and T. Wettig, Microscopic universality in the spectrum of the lattice Dirac operator, Phys. Rev. Lett. 80 (1998) 1146 hep-lat/9704018.

[24] R. Janik, New multicritical random matrix ensembles, Nucl. Phys. B 635 (2002) 492 hep-th/0201167. 